\title{
Selbstberichtete Praktiken von Lehrkräften im inklusiven Mathematikunterricht - Eine Interviewstudie
}

\author{
Susanne Prediger (iD - Sarah Buró
}

Eingegangen: 28. November 2019 / Angenommen: 15. Juli 2020 / Online publiziert: 13. August 2020

(C) Die Autorinnen 2020

Zusammenfassung Wie Lehrkräfte ihren inklusiven Mathematikunterricht ausgestalten, wird im Modell der gegenstandsbezogenen Expertise dadurch charakterisiert, welche Praktiken sie zur Bewältigung typischer didaktischer Anforderungssituationen nutzen: Lernvoraussetzungen identifizieren und diagnostizieren, differenzieren, fördern und gemeinsames Lernen anregen. Die qualitative Interviewstudie untersucht die selbstberichteten Praktiken der Lehrkräfte bzgl. der zugrundeliegenden inklusionsbezogenen Orientierungen und Wahrnehmungs- und Denkkategorien. Interviewt werden 17 Lehrkräfte, die ihre Praktiken beschreiben und reflektieren, und zwar alle zur gleichen Unterrichtseinheit „Prozente verstehen“ (Klasse 7). Die qualitative Analyse rekonstruiert fünf typische Leitkategorien: soziale Teilhabe, Beschäftigung, Aufgabenbewältigung, inhaltliche Teilhabe und stufengerechte Lernzuwächse, zwischen denen die Lehrkräfte wechseln. Die Analyse zeigt, wie sich diese Leitkategorien in den Praktiken genauer ausprägen.

Schlüsselwörter Inklusiver Unterricht · Inklusive Praktiken ·

Aufgabenbewältigung · Inhaltliche Teilhabe · Stufengerechte Lernzuwächse

MESC Codes 97 Mathematics Education

\section{Teachers' Self-reported Teaching Practices in Inclusive Mathematics Classrooms-An Interview Study}

\footnotetext{
Abstract Within the model of content-related expertise, we characterize how teachers realize inclusive education in mathematics classrooms by the teaching practices they enact to master typical didactical jobs: (1) identify potential demands and no-

S. Prediger $(\square)$

Deutsches Zentrum für Lehrerbildung Mathematik, Technische Universität Dortmund, Dortmund,

Deutschland

E-Mail: prediger@math.uni-dortmund.de
} 
tice students' abilities, (2) differentiate priorities, e.g. learning goals with respect to identified ability and compensate limited abilities by scaffolding, (3) enhance targeted learning goal with respect to ability, and (4) orchestrate joint learning. The qualitative interview study investigates teachers' self-reported practices with respect to the underlying inclusive orientations and categories for perception and thinking. 17 teachers were interviewed, they describe and reflect their practices within the same teaching unit on percentages in Grade 7. The qualitative analysis identifies five recurring leading categories: social participation, occupation, task propensity, content participation and adaptive learning progressions; teachers change between the five. The analysis shows how the leading categories are expressed in the different jobs and practices.

Keywords Inclusive education - Inclusive teaching practices · Task propensity · Academic participation · Adaptive learning progressions

\section{Einleitung}

Inklusiver Mathematikunterricht zielt auf nicht-aussondernden Unterricht (Wember 2013), der heterogene Lernvoraussetzungen aller Lernenden im gesamten Spektrum bis hin zu sonderpädagogischen Förderbedarfen berücksichtigt. Um ihn zu realisieren, bewältigen Lehrkräfte täglich vielfältige Anforderungssituationen: Sie diagnostizieren und fördern, differenzieren und moderieren gemeinsames Lernen (Korff 2015; Melzer et al. 2015; König et al. 2019). In einigen Studien wurden die Haltungen von Lehrkräften zum inklusiven Unterricht und ihre Einschätzung zur Relevanz dieser Anforderungssituationen untersucht (Melzer et al. 2015). Wenig ist jedoch dazu bekannt, mit welchen Praktiken deutsche Lehrkräfte die inklusionsbezogenen Anforderungssituationen bewältigen und vor welchem Hintergrund sie dies tun (Pool Maag und Moser Opitz 2014).

Zum Schließen dieser Forschungslücke trägt der vorliegende Artikel mithilfe einer Interviewstudie bei, in der Lehrkräfte über ihre Praktiken zum Bewältigen dieser Anforderungssituationen (in einer inklusiv aufbereiteten Unterrichtseinheit zum Prozentverständnis) berichten und reflektieren. Im Rahmen des Modells der gegenstandsbezogenen professionellen Expertise (Bromme 1992; Prediger 2019) werden die hinter diesen selbstberichteten Praktiken liegenden Orientierungen und Kategorien qualitativ rekonstruiert, um Rationalitäten der verschiedenen Praktiken $\mathrm{zu}$ verstehen und Anknüpfungspunkte für Fortbildungen $\mathrm{zu}$ finden.

Abschn. 2 des Artikels fasst die theoretischen Grundlagen und den derzeitigen Forschungsstand zusammen. Abschn. 3 stellt die herangezogenen Forschungsmethoden vor. Abschn. 4 berichtet Analyseergebnisse, die in Abschn. 5 kurz diskutiert werden. 


\section{Theoretischer Hintergrund}

\subsection{Anforderungssituationen an Lehrkräfte im inklusiven Mathematikunterricht}

In der Fachdidaktik wird für den inklusiven Unterricht eine Balance zwischen individuellem und gemeinsamen Lernen gefordert, indem die Einzelnen gemäß ihrer je spezifischen Profile gefördert und gemeinsames Lernen ermöglicht wird (vgl. Überblick in Scherer et al. 2016; Häsel-Weide und Nührenbörger 2017; in kritischer Auseinandersetzung auch Kollosche et al. 2019). Für Lehrkräfte ergeben sich daraus Anforderungen, die sich gemäß des Literaturüberblicks von Melzer et al. (2015) unterschiedlich strukturieren sowie relationieren lassen und neben dem Unterrichten auch vielfältige andere Bereiche umfassen. Bei Moser und Kropp (2014) werden etwa folgende sieben Anforderungsbereiche aus sonderpädagogischer Perspektive aufgeführt: Lernstands- und Entwicklungsdiagnostik, Beratung und Organisation, Lernund Entwicklungsförderung, binnendifferenzierte Unterrichtung, behinderungsspezifische Kommunikation, interdisziplinäre Kooperation und Förderung des sozialen Lernens. Bei König et al. (2019) werden Anforderungssituationen auf folgende vier verdichtet: Diagnostizieren, Intervenieren/Fördern, Management und Organisation, Beratung und Kommunikation. Da sich die letzten Doppel-Anforderungsbereiche auf außerunterrichtliche Anforderungen beziehen, werden sie von König et al. (2019) nicht weiter berücksichtigt; diesem Vorgehen folgt auch der vorliegende Artikel. Die ersten beiden werden hier aus fachdidaktischer Perspektive ausdifferenziert:

Im Projekt Matilda (Prediger et al. 2020) thematisieren wir vier didaktische Anforderungssituationen (drei eng verwobene zum individuellen Lernen, eine zum gemeinsamen Lernen), die im Folgenden knapp erläutert werden:

1. Lernvoraussetzungen identifizieren und diagnostizieren

2. Differenzierte Schwerpunkte setzen

3. Fokussiert fördern

4. Gemeinsames Lernen orchestrieren

Zu 1 Um inklusiven Unterricht adaptiv an Lernvoraussetzungen aller Lernenden anpassen zu können, sind relevante Lernvoraussetzungen zu identifizieren und diagnostizieren (Scherer und Moser Opitz 2010; Morris et al. 2009). Dazu gehören gemäß des Modells der individuellen Voraussetzungen erfolgreichen Lernens (INVOModell) von Hasselhorn und Gold (2009) neben dem Vorwissen (z. B. mathematische Verstehensgrundlagen und Basiskönnen sowie Sprachkompetenz) auch weitere kognitive Voraussetzungen (z. B. Aufmerksamkeitssteuerung und Arbeitsgedächtnis) und motivational-volitionale Voraussetzungen (z. B. Motivation und metakognitive Regulation). Diese Vielfalt potenziell relevanter Lernvoraussetzungen erfordert bei Lehrkräften vielfältige Diagnosekategorien: Je nachdem welche Lernvoraussetzungen identifiziert und diagnostiziert werden, werden verschiedene weitere Praktiken genutzt.

Zu 2 Um den heterogenen Lernvoraussetzungen einer inklusiven Lerngruppe gerecht zu werden, muss Unterricht adaptiv gestaltet werden. „Adaptiv“ wird hier de- 
finiert als an individuelle Voraussetzungen angepasst, indem differenzierte Schwerpunkte gesetzt werden (Lawrence-Brown 2004). Die Anpassung kann durch zwei komplementäre Strategien erfolgen (Wember 2013): In Unterstützungsstrategien werden fehlende Lernvoraussetzungen durch Entlastung oder Unterstützung umgangen. Beispielsweise kann ein begrenztes Arbeitsgedächtnis durch übersichtliche Arbeitsblattgestaltung oder schwache metakognitive Regulation durch stärkere Vorstrukturierung entlastet werden (Krajewski und Ennemoser 2010). In Förderstrategien dagegen werden fehlende Lernvoraussetzungen direkt gefördert, um Anschlussfähigkeit an spätere Anforderungen zu erzielen (Wember 2013). Dies ist sowohl bzgl. einiger sprachlicher Anforderungen als auch bzgl. zentraler Elemente des mathematischen Vorwissens maßgeblich, denn Wissenszuwachs in der Mathematik ist hochkumulativ. Das Setzen differenzierter Schwerpunkte umfasst daher insbesondere das Festlegen differenzierter Lernziele auf einer gut durchdachten Lernstufung (Prediger und von Aufschnaiter 2017; Krähenmann et al. 2019). Zusammenfassend kann also das Setzen individueller Schwerpunkte in kurzfristiger Perspektive als Unterstützen durch Umgehen möglicher Schwierigkeiten erfolgen und in langfristiger Perspektive als Fördern, um Lernzuwächse zu erzielen (s. unten). Entsprechend wird eher durch Anforderungsstufung (durch steigende Kompliziertheit, Vorstrukturierung o. ä.) oder durch Lernstufung (mit Differenzierung nach Lernzielen, vgl. Prediger und von Aufschnaiter 2017) differenziert. Beides kann in Praktiken der offenen Differenzierung umgesetzt werden (auch Selbstdifferenzierung/natürliche Differenzierung genannt), bei der Lernende aus einem Spektrum von Lern- und Anforderungsstufungen die Herausforderungen selbst wählen (Scherer et al. 2016; Tomlinson 2001), oder durch geschlossene Differenzierung, bei der Lehrkräfte gezielt (ggf. diagnosegeleitet) Anforderungen zuweisen.

Zu 3 Die Jobbeschreibung Fokussierte Förderung wurde als Alternativbegriff zu Individueller Förderung etabliert, um falsche Assoziationen von Eins-zu-Eins-Betreuung oder methodisch voll individualisiertem Unterricht zu vermeiden (Prediger 2016; Pool Maag und Moser Opitz 2014). Fokussierte Förderung zielt dagegen nicht allein auf die Sozialform, sondern wird durch zwei zentrale Qualitätskriterien charakterisiert: (a) Adaptivität im zuvor definierten Sinne und (b) fachdidaktische Treffsicherheit der Förderinhalte. Für mathematisch schwache Lernende (mit und ohne diagnostizierten sonderpädagogischen Förderbedarf) bedeutet dies insbesondere, nicht nur Kalküle zu fokussieren, sondern das Verstehen der mathematischen Inhalte (Beswick 2007; Boyd und Bargerhuff 2009), also neben einem prozeduralen Basiskönnen (z.B. additive Zerlegungen der 10) auch Verstehensgrundlagen der vorangehenden Jahrgänge (z. B. Multiplikationsverständnis) adaptiv zu fördern (Scherer und Moser Opitz 2010; Prediger 2016). Wie der Empirieteil dieses Artikels zeigen wird, sind die realisierten Förderpraktiken von Lehrkräften deutlich breiter und oft nicht fokussiert im hier definierten Sinne.

Zu 4 Die Forderung im inklusiven Fachunterricht gemeinsames Lernen zu gestalten, ist Kern des Inklusionsgedankens. Dieser wird jedoch sehr unterschiedlich interpretiert und ausgestaltet (Überblick über vielfältige Varianten in Kollosche et al. 2019). Sie hat sowohl eine soziale Dimension im Sinne des sozialen Miteinan- 
ders als auch eine inhaltliche Dimension, die die Gemeinsamkeit auf das fachliche Lernen bezieht (Wocken 1998; Häsel-Weide und Nührenbörger 2017; König et al. 2019). Dabei wird ein Spektrum von Miteinander-Lernen (z.B. als gemeinsames Lernen an gleichen Inhalten im Gleichschritt, so lange dies möglich ist) bis Voneinander-Lernen (bei dem Lernende auf verschiedenen Lernstufen mit gegenseitiger Bereicherung kommunizieren) beschrieben. Das Verb „orchestrieren“ drückt dabei die didaktische Anforderung aus, mehrere Lernwege gleichzeitig anzuleiten und zueinander in Beziehung zu setzen.

Insgesamt hat die fachdidaktische Entwicklung und Forschung auf Unterrichtsebene also verschiedene produktive Praktiken für die Bewältigung der Anforderungssituationen entwickelt. Sie bilden den präskriptiven Hintergrund, vor dem sich die von Lehrkräften berichteten vielfältigen (produktiven und weniger produktiven) Praktiken einordnen lassen.

\subsection{Modell der gegenstandsbezogenen professionellen Expertise als theoretischer Rahmen zur Charakterisierung von selbstberichteten Praktiken}

Um Praktiken von Lehrkräften zu beschreiben und bzgl. ihrer Hintergründe zu charakterisieren, wird auf das gegenstandsbezogene Expertisemodell zurückgegriffen, das sich an Bromme (1992) anlehnt und für gegenstandsbezogene Professionalisierungsforschung adaptiert wurde (Prediger 2019). In diesem Modell erfolgt die Konzeptualisierung von Lehrkräfte-Expertise ausgehend von typischen Anforderungssituationen (Prediger 2019):

- Didaktische Anforderungssituationen (abgekürzt auch Jobs genannt) sind typische Konstellationen im Unterrichtsgeschehen, die eine Lehrkraft zur Realisierung des (hier inklusiven) Unterrichts bewältigen muss.

- Praktiken sind definiert als wiederkehrende Handlungsmuster zur Bewältigung didaktischer Anforderungssituationen. Sie sind charakterisierbar durch die dazu genutzten didaktischen Werkzeuge sowie die zugrundeliegenden Orientierungen und Kategorien (s. unten).

- Zu den didaktischen Werkzeugen gehören alle Artefakte und Instrumente, mit denen Lehrkräfte Unterricht gestalten, z.B. Aufgaben, Methoden, Gesprächsführungsimpulse oder Unterstützungsformate. Sie werden zu didaktischen oder pädagogischen Werkzeugen, wenn sie zu unterrichtlichen Zwecken eingesetzt werden.

- Orientierungen sind gegenstandsübergreifende oder -bezogene Haltungen, die die Priorisierungen und Interpretationen der Lehrkräfte zur jeweiligen Anforderungssituation implizit oder explizit prägen und damit den Praktiken zugrunde liegen. Darunter fallen z. B. generelle Haltungen zum Unterrichtsgegenstand oder zu Lernprozessen, aber auch individuelle Relevanzsetzungen, was wertvollen inklusiven Unterricht ausmacht.

- Denk- und Wahrnehmungskategorien betreffen das konzeptuelle, nicht-propositionale Wissen, das die kategorialen Wahrnehmungs- und Denkweisen der Lehrkräfte filtert und so ihre Praktiken fokussiert. Kategorien bilden jeweils ein kom- 
plexes Geflecht, bei denen einige als Leitkategorien besonders stark das Wahrnehmen, Handeln und Reflektieren von Praktiken (und damit auch ihre reflektierte Weiterentwicklung) prägen (Prediger 2020).

Im Rahmen des gegenstandsbezogenen Expertisemodells wird in diesem Artikel empirisch rekonstruiert, welche Praktiken Lehrkräfte zum Bewältigen der zuvor spezifizierten Anforderungssituationen berichten, und welche Orientierungen und Kategorien sie dabei explizit oder implizit zugrunde legen (Prediger 2019). Diese Jobanalyse gilt ,als Heuristik bei der Suche nach den ,natürlichen“ Kategorien des Expertenwissens" (Bromme 1992, S. 88).

\subsection{Inklusionsbezogene Orientierungen von Lehrkräften}

In Bezug auf inklusionsbezogene Orientierungen identifiziert die internationale pädagogische und sonderpädagogische Forschungsliteratur bislang vor allem eine (Nicht-)Akzeptanz zur Inklusion und zeigt dazu unterschiedliche Ergebnisse je nach Schulsystem, Vorerfahrungen und Behinderungsformen (vgl. den Survey von de Boer et al. 2011). Doch nicht nur die generelle (Nicht-)Akzeptanz prägt Entscheidungen und Praktiken von Lehrkräften im inklusiven Unterricht, sondern der Meta-Analyse von Scruggs und Mastropieri (1996) folgend auch spezifischere Orientierungen.

Spezifischere Orientierungen wurden in der Forschungsliteratur zum Denken von (angehenden und praktizierenden) Lehrkräften über inklusiven Mathematikunterricht identifiziert, z. B. zu didaktischen Umsetzungsqualitäten (Korff 2015; Pool Maag und Moser Opitz 2014) oder zum Umgang mit schwachen oder benachteiligten Lernenden (Beswick 2007; Wilhelm et al. 2017). Auch wenn diese Studien unterschiedliche Konzeptualisierungen von Einstellungen, Haltungen, Überzeugungen und Orientierungen nutzen, lassen sich aus ihnen und aus eigenen Vorstudien (Büscher 2019; Prediger et al. 2016; Prediger 2020) vier Orientierungen herausarbeiten, die bzgl. der in Abschn. 2.1 spezifizierten Anforderungssituationen relevant sein könnten:

- Soziale Orientierung im inklusiven Unterricht: Einer sozialen Orientierung folgen Lehrkräfte, die soziale Teilhabe (also das Miteinander aller Lernenden in der Klassengemeinschaft) fördern. Dies umfasst nach Koster et al. (2009) Beziehungen, positive Kontakte und Interaktionen ohne Ausgrenzung in Freizeit- und Unterrichtssituationen, die Anerkennung der Andersartigkeit durch Peers und die eigene Wahrnehmung von Akzeptanz. Wenn soziale Orientierung mit inhaltlicher Orientierung verknüpft wird, wird zusätzlich inhaltliche Teilhabe angestrebt (s. unten).

- Inhaltliche Orientierung im inklusiven Mathematikunterricht: Die inhaltliche Orientierung bezieht sich auf die grundlegend fachdidaktische Orientierung des Lehrkräftehandelns an fachlichen Lern- und Arbeitsprozessen der Schülerinnen und Schüler. Sie ist nicht automatisch eine handlungsleitende Orientierung. So bilanziert z. B. Martens (2018) in seinem Forschungsüberblick zum individualisierten Unterricht, dass in vielen empirisch rekonstruierten Praktiken wenig inhaltliche Orientierung am Lernen der Kinder erfolgt, sondern nur z. B. Arbeitsverhalten oder -tempo fokussiert wird. Korff (2015) resümiert in ihrer Interviewstudie zu 
inklusiven Praktiken im Mathematikunterricht der Grundschule, dass einige Lehrkräfte für Lernende mit diagnostiziertem sonderpädagogischen Förderbedarf die inhaltliche Orientierung mit anderen Qualitätsmaßstäben versehen (z. B. durch Reduktion auf Beschäftigung ohne inhaltliche Ansprüche) als für Lernende ohne sonderpädagogischen Förderbedarf. Auch Befunde, dass Lehrkräfte für schwächere oder sozial benachteiligte Lernende geringe fachliche Erwartungen und wenig kognitiv anspruchsvolle Lerngelegenheiten vorsehen (Beswick 2007; Wilhelm et al. 2017), lassen sich als nicht priorisierte inhaltliche Orientierung beschreiben. Eine durchgängige inhaltliche Orientierung auf fachliches Lernen dagegen schafft für alle Lernenden reichhaltige und auf der jeweiligen Lernstufe anspruchsvolle Lerngelegenheiten (Pool Maag und Moser Opitz 2014). Im inklusiven Fachunterricht sind inhaltliche und soziale Orientierung nicht als Gegensätze zu verstehen, sondern spannen zwei Dimensionen auf, die im Idealfall produktiv kombiniert werden, wenn das soziale Miteinander auch auf inhaltliche Teilhabe bezogen wird, z. B. im Streben nach Voneinander-Lernen (Häsel-Weide und Nührenbörger 2017; Korff 2015). Empirisch weiter auszudifferenzieren ist, wie sich Kombinationen von sozialer und inhaltlicher Orientierung bei Lehrkräften ausdrücken können.

- Verstehens- vs. Kalkülorientierung: Innerhalb der inhaltlichen Orientierung betrifft der am besten untersuchte Teilaspekt im inklusiven Unterricht die Verstehens- und Kalkülorientierung: Verstehensorientierung leitet das Handeln von Lehrkräften, wenn sie den Aufbau von konzeptuellem Verständnis anstreben. Kalkülorientierung dagegen zielt auf den Aufbau von Rechenkalkülen und Faktenwissen. Während gerade für mathematisch Schwache die Relevanz des Aufbaus von konzeptuellem Verständnis betont wird (Scherer und Moser Opitz 2010), zeigen viele Untersuchungen bei Lehrkräften eine starke Kalkülorientierung (Beswick 2007; Boyd und Bargerhuff 2009; Korff 2015). Einige Lehrkräfte beziehen die starke Kalkülorientierung auf alle Lernenden, insbesondere bei generell prozeduralem Mathematikbild. Andere beziehen Kalkülorientierung vorrangig auf mathematisch Schwache, dann ist sie als wichtige Ausprägung der geringen Erwartungen an diese Gruppe zu verstehen (Beswick 2007; Wilhelm et al. 2017; Büscher 2019).

- Langfristigkeit vs. Kurzfristigkeit: Als weitere Gelingensbedingung zur inhaltlichen Orientierung haben Prediger et al. (2016) sowie Gravemeijer et al. (2016) eine langfristige statt kurzfristige Orientierung identifiziert: Praktiken in kurzfristiger Orientierung fokussieren auf die inhaltliche Bewältigung der einzelnen Situation. Praktiken in langfristiger Orientierung nehmen dagegen Lernprozesse über ganze Unterrichtseinheiten oder länger in den Blick. Gravemeijer et al. (2016) beschreiben ,task propensity“ als eine auf Kurzfristigkeit ausgerichtete Tendenz vieler Lehrkräfte, einzelne Aufgaben lediglich bzgl. der Möglichkeit zu betrachten, wie Lernende sie bewältigen, nicht jedoch bzgl. des Potenzials, zu einer längerfristigen Lernstufung beizutragen. In Bezug auf Differenzierung haben Prediger und von Aufschnaiter (2017) den Unterschied als Differenzieren nach Anforderungsstufung oder nach Lernstufung beschrieben. Bzgl. Förderung äußert sich die kurzfristige Orientierung in dem ausschließlichen Einsatz von Unterstützungsstrategien durch Anforderungsreduktion statt durch Förderstrategien (s. oben). Die von Wilhelm et al. (2017) in Unterrichts-Videostudien rekonstruierten Unterfor- 


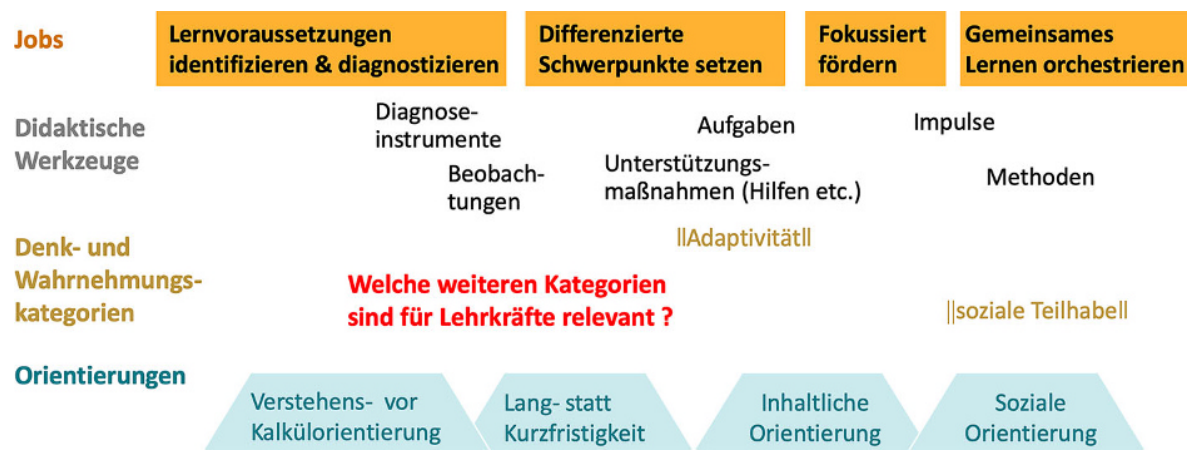

Abb. 1 Zusammenfassung der relevanten Komponenten im Modell für inklusionsbezogene Expertise und Verortung der Forschungsfrage. (Bildrechte bei S. Prediger)

derungspraktiken lassen sich mit dieser Unterscheidung so fassen: Anforderungsreduktionen ermöglichen in kurzfristiger Orientierung für Lehrende und Lernende Erfolgserlebnisse, wenn die Aufgabenbewältigung der Maßstab ist. Sie sind also in dieser Orientierung effektiv. Dass sie allerdings nicht zu mittel- oder langfristigen Lernfortschritten beitragen, bedeutet in langfristiger Orientierung einen Entzug von Lerngelegenheiten (Prediger 2020; Gravemeijer et al. 2016).

Auch wenn diese Orientierungen einzeln bereits empirisch dokumentiert sind, ist ihr Zusammenspiel für die verschiedenen Jobs des inklusiven Fachunterrichts bislang nicht untersucht.

\subsection{Zusammenfassung und Forschungsfrage}

Zusammenfassend lässt sich festhalten: Auch wenn die Forschung zu Jobs und Orientierungen $\mathrm{zu}$ inklusivem Mathematikunterricht bei weitem nicht einheitlich ist (Kollosche et al. 2019; Scherer et al. 2016), können dennoch relevante Komponenten für ein Modell inklusionsbezogener professioneller Expertise extrahiert werden. Insbesondere lassen sich bereits einige Orientierungen identifizieren, die die Praktiken der Lehrkräfte zur Bewältigung von vier zentralen Jobs leiten können. Diese wurden für die vorliegende Studie in Abb. 1 zusammengestellt (in den ersten zwei Zeilen der Abb. 1 steht zusammengehöriges untereinander, die Orientierungen sind in jedem Job relevant).

Wenig ist jedoch darüber bekannt, wie die Praktiken zur Bewältigung verschiedener Jobs zusammenhängen und welche Orientierungen und Kategorien den Praktiken im Denken der Lehrkräfte jeweils zugrunde liegen. Die Interviewstudie bearbeitet daher folgende Forschungsfrage:

Durch welche Kategorien lassen sich die Lehrkräfte beim Berichten und Reflektieren ihrer Praktiken zur Bewältigung verschiedener inklusionsbezogener Anforderungssituationen leiten? 


\begin{tabular}{|c|c|c|c|c|c|c|}
\hline \multicolumn{2}{|c|}{$\begin{array}{l}\text { Relevante Verstehengrund- } \\
\text { lagen (V) \& Basiskönnen (B) }\end{array}$} & \multirow{3}{*}{$\begin{array}{l}\text { Kompetenzen }(\mathrm{K}) \text { als Lern- } \\
\text { stufen der Unterrichtsreihe } \\
\text { K1 Prozente und Brüche ab- } \\
\text { schätzen und darstellen }\end{array}$} & \multirow{2}{*}{$\begin{array}{l}\text { Thema } \\
\text { Einführung Streifen } \\
\text { Anteile schätzen }\end{array}$} & \multirow{2}{*}{$\begin{array}{l}\begin{array}{l}\text { Aufgaben im } \\
\text { Basisniveau }\end{array} \\
1.2\end{array}$} & \multirow{2}{*}{$\begin{array}{l}\text { Gemeinsame } \\
\text { Aufgaben } \\
1.1\end{array}$} & \multirow{2}{*}{$\begin{array}{l}\text { Aufgaben im } \\
\text { Regelniveau } \\
1.2\end{array}$} \\
\hline & Anteil-Ganzes-Konzept & & & & & \\
\hline & & & Verknüpfen mit Brüchen & - & & $1.3,1.4$ \\
\hline & $\begin{array}{l}\text { Multiplizieren als Zăhlen } \\
\text { in Schritten und }\end{array}$ & \multirow{3}{*}{$\begin{array}{l}\text { Prozentwerte und -sätze } \\
\text { am Streifen finden }\end{array}$} & Bezug der Skalen herstellen & & $2.1,2.2$ & \\
\hline & Dividieren als Passen-in & & Zählen in doppelten Schritten & & $2.3,2.4$ & \\
\hline \multirow[t]{2}{*}{ B4 } & Zerlegen der $100,60,80$, & & Formale Päckchen & - & & 2.5 \\
\hline & $\begin{array}{l}\cdots \\
\text { Umwandeln Prozente - }\end{array}$ & \multirow[t]{4}{*}{$\begin{array}{l}\text { K3 Prozentwerte und -sätze } \\
\text { bestimmen }\end{array}$} & $\begin{array}{l}\text { Hoch- und Runterrechnen } \\
\text { von Prozentwerten und -sätzen }\end{array}$ & & 3.1 & 3.2 \\
\hline \multirow{3}{*}{$\begin{array}{l}\text { V6 } \\
\text { B7 }\end{array}$} & einfache Brüche & & $\begin{array}{l}\text { Sprachspeicher für bedeutungs- } \\
\text { bezogene Vokabeln }\end{array}$ & & $\begin{array}{l}3.3 \\
3.4\end{array}$ & \\
\hline & Zwei Skalen verknüpfen & & Üben des Hoch- \& Runterrechnens & & $3.5,3.7$ & 3,8 \\
\hline & $\begin{array}{l}\text { Zählen in doppelten } \\
\text { Schritten }\end{array}$ & & Einführung formalbezogener Vokabeln & & 3.6 & \\
\hline \multirow[t]{2}{*}{ V8 } & Proportionales Hoch- & \multirow[t]{2}{*}{$\begin{array}{l}\text { K4 Grundwerte am Streifen } \\
\text { finden und bestimmen }\end{array}$} & $\begin{array}{l}\text { Typ Grundwert gesucht } \\
\text { Hoch- und Runterrechnen }\end{array}$ & $4.2,4.4,4.5$ & $\begin{array}{l}4.1 \\
4.3\end{array}$ & $4.2,4.4$ \\
\hline & & & Verknüpfung mit Dreisatztabelle & - & & 4.5 \\
\hline & & \multirow{2}{*}{$\begin{array}{l}\mathrm{K}_{\mathrm{R}} \text { Umgang mit } \\
\text { Verminderung }\end{array}$} & Sensibilisieren für sprachliche Details & - & & $5.1,5.2$ \\
\hline \multirow{4}{*}{\multicolumn{2}{|c|}{$\begin{array}{l}\text { Strategien zur meta- } \\
\text { kognitiven Regulation }\end{array}$}} & & Grundwerte nach Verminderung & - & & $5.3,5.4$ \\
\hline & & $\begin{array}{c}\mathrm{K} 5_{B} / 6_{R} \text { Verschiedene Text- } \\
\text { aufgaben unterscheiden }\end{array}$ & $\begin{array}{l}\text { Grundaufgaben erkennen und ggf. } \\
\text { abweichende Strukturen erfassen }\end{array}$ & & $\begin{array}{l}5.1_{B}=6.1_{R} \\
5.2_{B}=6.3_{R} \\
5.3_{B}=6.5_{R}\end{array}$ & $\begin{array}{l}6.2{ }^{*}{ }_{R} \\
6.4{ }^{*}{ }_{R} \\
6.6{ }^{*}{ }_{R}\end{array}$ \\
\hline & & $\begin{array}{l}\mathbf{K 7 _ { R }} \text { Textaufgaben selbst } \\
\text { erstellen }\end{array}$ & Textaufgaben selbst stellen & & $\begin{array}{l}\text { Aufgaben } \\
\text { selbst stellen }\end{array}$ & $7.1,7.2,7.3$ \\
\hline & & $\begin{array}{l}\mathrm{K} 8_{\mathrm{R}} \text { Schwierige Textaufgaben } \\
\text { bearbeiten }\end{array}$ & $\begin{array}{l}\text { Am Prozentstreifen komplexe } \\
\text { Textaufgaben entschlüsseln }\end{array}$ & - & & $\begin{array}{l}8.1,8.2,8.3 \\
8.4\end{array}$ \\
\hline
\end{tabular}

Abb. 2 Übersicht zur Unterrichtsreihe Prozente verstehen (Pöhler et al. 2018). (Bildrechte bei S. Prediger)

\section{Methoden der Datenerhebung und -auswertung}

\subsection{Forschungskontext der Interviewstudie}

Die hier berichtete Interviewstudie ist eine Teilstudie des BMBF-geförderten Professionalisierungsforschungsprojekts Matilda (,Mathematik inklusiv lehren lernen“), in dem die Professionalisierungswege von Lehrkräften zum inklusiven Unterricht gestaltet und untersucht werden (Prediger et al. 2020). Kombiniert wird dazu DesignResearch auf Unterrichts- und Fortbildungsebene mit einer qualitativen Interviewstudie, einer Unterrichtsvideostudie und einer quantitativen Wirksamkeitsstudie im Prä-Post-Kontrollgruppen-Design (Prediger et al. 2020).

Alle Teilstudien erfolgen exemplarisch an der inklusiven Unterrichtseinheit „Prozente verstehen“ in Klasse 7 (Pöhler et al. 2018). Die Unterrichtsreihe (Abb. 2) zeichnet sich durch eine starke Verstehensorientierung aus, die Förderstrategien für Verstehensgrundlagen und zu automatisierendes Basiskönnen systematisch in das Material einbezieht (Abb. 2 linke Spalte, z. B. V3 Multiplizieren als Zählen in Schritten und B4 Zerlegen der 100). Das Material differenziert in Basis- und Regelfassungen mit vielfältigen, selbstdifferenzierenden Aufträgen (Lawrence-Brown 2004). Etwa $80 \%$ der Aufgaben (nummeriert mit 1.1 bis 8.4) der Basisfassung sind auch in der Regelfassung enthalten, um gemeinsames Lernen in Kombination aus sozialer und inhaltlicher Orientierung zu ermöglichen. Die Explizierung von zu erwerbenden Kompetenzen K1-K8 in einer Checkliste sowie der Verstehensgrundlagen und des Basiskönnens soll bei Lehrkräften ein Denken in Lernstufungen unterstützen. 


\subsection{Methoden der Datenerhebung}

Die methodische Herangehensweise der Interviewstudie wurde gewählt, auch wenn aus anderen Studien bekannt ist, dass selbstberichtete Praktiken nicht immer den real umgesetzten Praktiken entsprechen (Mullens und Gayler 1999). Doch elizitiert das halbstrukturierte Interview die Berichte und Erklärungen der Lehrkräfte zu ihren Praktiken, die mehr Aufschluss über die (zur Planung, Durchführung und Reflexion herangezogenen) Denk- und Wahrnehmungskategorien sowie Orientierungen geben als eine Unterrichtsbeobachtungsstudie. Trotz einer zu berücksichtigenden Gefahr sozial erwünschter Antworten, erlaubt die Interviewform die Rekonstruktion derjenigen Kategorien, die das Denken der Lehrkräfte leiten (Mullens und Gayler 1999). Die Passung zwischen selbstberichteten und tatsächlich realisierten Praktiken wird in einer späteren Teilstudie untersucht, indem die Befunde mit denen der noch laufenden Unterrichtsbeobachtungsstudie in Beziehung gesetzt werden.

Für die Interviewstudie wurden mit 17 Lehrkräften sogenannte Planungsgespräche in Gruppen von ein bis vier Personen geführt, die jeweils 30-75 min dauerten. Um möglichst unterschiedliche Ansätze zu erfassen, wurden Lehrkräfte nach variierender Unterrichtserfahrung allgemein (zwischen 1 und 18 Jahren) und im inklusiven Unterricht (zwischen 0 und 3 Jahren) ausgewählt. 14 der 17 Lehrkräfte hatten eine mathematikdidaktische, 2 Lehrkräfte eine sonderpädagogische Ausbildung und eine hatte beide Ausbildungen. Alle 17 Lehrkräfte planten ihren Unterricht mit dem inklusiven Unterrichtsmaterial Prozente verstehen (Abb. 2), worunter sieben auch Fortbildungen zu Aspekten aus Abschn. 2.1 und 2.3 besuchten (Details in Prediger et al. 2020). Zum Zeitpunkt der Planungsgespräche hatten die Lehrkräfte bereits 1-8 (von insgesamt ca. 16) Stunden der Unterrichtseinheit unterrichtet.

Der Leitfaden des halbstrukturierten Interviews adressierte die Praktiken der Lehrkräfte zu allen vier Anforderungssituationen in sieben Themenblöcken: Beginn der Reihe, Auswahl der Materialversion, Balance des gemeinsamen und individuellen Lernens, Reflexion über die bisherigen Stunden, gesetzte Lernziele, individuelle Definition für ,gelungenen“ inklusiven Unterricht sowie abschließend offene Fragen und Impulse zur Materialüberarbeitung.

\subsection{Methoden der Datenauswertung}

Die insgesamt 497 min umfassenden Aufnahmen der 17 Interviews wurden vollständig transkribiert. Das qualitative Analyseverfahren basiert auf dem (in Abschn. 2.2 vorgestellten) Modell der gegenstandsbezogenen professionellen Expertise, um die Praktiken der Lehrkräfte für verschiedene Anforderungssituationen zu erfassen und die dabei zugrundeliegenden Orientierungen und Kategorien zu rekonstruieren. Die in der existierenden Forschung bereits gut dokumentierten Orientierungen wurden dazu deduktiv kodiert, die relevanten Denk- und Wahrnehmungsschritte erst induktiv in einem kategorienbildenden Verfahren gewonnen. Kombiniert wurden dazu vier Auswertungsschritte (Mayring 2015):

1. Alle Äußerungen wurden in Sinneinheiten gegliedert. Die Sinneinheiten wurden bzgl. der jeweils rekonstruierbaren Orientierungen und adressierten Jobs deduktiv 
kodiert (statt fokussiert fördern wurde fördern kodiert, weil die berichteten Praktiken nur manchmal fokussiert sind) und durch die angesprochenen didaktischen Werkzeuge (z. B. Beobachtungen, Tests, differenzierte Aufgaben, Unterstützungsformate usw.) charakterisiert. Insgesamt wurden 1099 Codes für Jobs vergeben. Orientierungen wurden dabei gemäß dem Modell der gegenstandsbezogenen professionellen Expertise (Prediger 2019) mit <...> markiert, um jeweils strukturelle Transparenz zu schaffen.

2. Die durch diese Kodierung grob erfassten Praktiken zu den verschiedenen Jobs wurden im zweiten Schritt auf die zugrundeliegenden Wahrnehmungs- und Denkkategorien untersucht. In einem kategorienentwickelnden Vorgehen mit systematischen Fallvergleichen wurden die bei den Lehrkräften rekonstruierbaren Kriterien in musterhaft wiederkehrende Kategorien zusammengefasst und hinsichtlich zugrundeliegender Orientierungen charakterisiert. So konnten fünf übergeordnete Leitkategorien und einige weitere Kategorien induktiv identifiziert werden, die die inklusionsbezogenen Praktiken der Lehrkräfte zu leiten scheinen. Kategorien werden gemäß dem Modell mit II...I markiert (Prediger 2019). Im übergreifenden Projekt wurden die identifizierten Kategorien kommunikativ validiert und mit weiteren Datenquellen trianguliert (z. B. Videos aus Fortbildungen und Unterricht).

3. Mit den identifizierten Kategorien wurden alle rekonstruierten Praktiken analysiert.

4. Mögliche individuelle Relevanzsetzungen wurden durch die Häufigkeiten der adressierten Leitkategorien in den unterschiedlichen Anforderungssituationen bestimmt. Gezählt wurden dabei die selbstgetätigten Aussagen in Sinneinheiten, aber nicht Kurzäußerungen wie „Ja, genau“ oder „Mhmm“. Die ermittelten Häufigkeiten geben eine erste Annäherung an unterschiedliche Schwerpunktsetzungen, sind jedoch mit Vorsicht zu interpretieren, da sie z.B. auch vom Gesprächsverlauf geprägt sein könnten.

\section{Empirische Einsichten in berichtete Praktiken und ihre Hintergründe}

In Abschn. 4.1 werden die rekonstruierten zentralen Denk- und Wahrnehmungskategorien (kurz Leitkategorien) an Transkriptbeispielen vorgestellt. In Abschn. 4.2 wird verglichen, wie sie die Praktiken zu den verschiedenen Jobs prägen. Zur Verdeutlichung, dass Lehrkräfte jeweils mit mehreren Leitkategorien arbeiten und verschieden kohärent zwischen Jobs verknüpfen, gibt Abschn. 4.3 abschließend einen Überblick zu den Häufigkeiten. Eine kompakte Zusammenfassung der rekonstruierten Zusammenhänge zeigt Abschn. 5.1.

\subsection{Fünf wiederkehrende Leitkategorien}

In Schritt 3 der Datenanalyse wurden die von Lehrkräften geäußerten Kriterien zu insgesamt fünf Leitkategorien zusammengefasst, die das Wahrnehmen und Denken leiten: \|soziale Teilhabel, \|Beschäftigung\|, \|Aufgabenbewältigung\|, \|inhaltliche Teilhabell und Ilstufengerechte Lernzuwächsell. $\mathrm{Zu}$ jeder Leitkategorie wird im 
Folgenden ein kurzes Fallbeispiel gegeben und eine kurze Charakterisierung vorgenommen, auf welchen Orientierungen sie jeweils beruhen.

\section{Leitkategorie: Soziale Teilhabe}

Die Leitkategorie Ilsoziale Teilhabell aktivieren Lehrkräfte, wenn sie das soziale Miteinander aller Lernenden fokussieren; sie basiert stets auf einer <sozialen Orientierung>. Sie kann mit anderen Kategorien und Orientierungen verknüpft oder isoliert auftreten. Zwei typische Äußerungen zur sozialen Teilhabe werden in Transkript 1 abgedruckt.

\section{Transkript 1: Quinn Tacke}

Kontext: Was ist gelungener inklusiver Unterricht?

165a Quinn Gelungen ist er dann, wenn die inklusiven Schüler möglichst lange da- || ||soziale Teilhabe\| oder ran teilhaben können an dem Unterricht. $\|$ inhaltliche Teilhabe $<$ soziale Orientierung $>$

165b Quinn Indem sie zwar andere Aufgaben bearbeiten, aber zu einem gleichen Ergebnis kommen und sich auch melden können und sich zugehörig fühlen. Ich glaube, das Schlimmste, was passieren kann, ist, dass die sich abgehängt fühlen und merken: „Ich gehöre gar nicht mehr dazu.“

Job Schwerpunkte setzen ||soziale Teilhabe\| ||Aufgabenbewältigung $\|$

Die erste Äußerung in Turn 165a ist ein Beispiel, in dem die Kategorie ITeilhabell explizit formuliert wird. Quinns Äußerung ,,möglichst lange daran teilhaben können“ (Turn 165a) allein macht jedoch noch nicht deutlich, ob hier eine rein <soziale Orientierung $>$ zugrunde liegt und somit rein Ilsoziale Teilhabell im Sinne des zwischenmenschlichen Miteinanders gemeint ist, oder ob dem „daran“ zusätzlich auch eine $<$ inhaltliche Orientierung > zugrunde liegt. Dann würde auch die Kategorie llinhaltliche Teilhabell rekonstruiert, die <inhaltliche und soziale Orientierungen> verknüpft (s. unten). Quinns zweite Äußerung in Turn 165b ist ein Beispiel, in dem sich beim Job Differenzierte Schwerpunkte setzen die Kategorie Ilsoziale Teilhabell als expliziertes (wenn auch nicht wörtlich angesprochenes) Kriterium (,sich zugehörig fühlen") verselbständigt. Ihr wird die inhaltliche Kategorie \|Aufgabenbewältigung\| (,sie zwar andere Aufgaben bearbeiten, aber zu einem gleichen Ergebnis kommen und sich auch melden können“) untergeordnet. Das Erreichen desselben Ergebnisses dient nur <kurzfristig $>$ dem Zugehörigkeitsgefühl in <sozialer Orientierung $>$, nicht zuvorderst einem Lernziel in <inhaltlicher Orientierung $>$.

\section{Leitkategorie: Beschäftigung}

Die Leitkategorie \|Beschäftigung\| nutzen Lehrkräfte implizit oder explizit, wenn ihre Praktiken darauf ausgerichtet sind, Lernende zur aktiven Arbeit im Unterricht zu bewegen. Sie basiert nicht auf einer expliziten <inhaltlichen Orientierung $>$ an den konkreten Lerninhalten, sondern ist meist in <kurzfristiger Orientierung $>$ im Sinne eines Einstiegs oder Durchhaltens in einer Arbeitsphase gedacht. Eine Beispieläußerung zur \|Beschäftigung\| ist etwa folgende (Transkript 2). 


\section{Transkript 2: Ulla Kaiser}

Kontext: Die starken Lernenden arbeiten in kooperativen Lernformen, für die Förderkinder gilt das nicht.

70a Ulla Die leistungsschwachen Schüler, ähm, die sind ganz oft einfach raus. Also die sind, die machen dann einfach gar nichts. Wir haben einen Schüler, der hat - gestern ist eine andere Kollegin noch mit reingekommen.

70b Ulla Die hat dann wirklich nur mit dem Schüler an dem Material gearbeitet, aber das muss dann wirklich ganz klar für den, ähm, nochmal zusammengefasst werden und der $[\ldots]$ durchbegleitet werden $[\ldots]$
Job Identifizieren/Diag-

nostizieren

||Beschäftigung ||

$<$ kurzfristig $>$

\section{Job Fördern}

keine \|inhaltlichen Lernziele $\|$ expliziert

$<$ kurzfristig $>$

Ulla diagnostiziert, dass ihre schwächeren Lernenden nicht aktiv arbeiten (,die machen dann einfach gar nichts“). Die (in dieser Klasse nur seltene) Förderressource der Doppelbesetzung investiert sie daher in $\|$ Beschäftigung\|, d.h. dafür, dass der Schüler überhaupt aktiv bleibt (,muss ... durchbegleitet werden“). Dass beim Fördern auch Ilinhaltliche Lernzielell oder IAufgabenbewältigung\| angestrebt wurden, könnte in dem „nochmal zusammengefasst“ angedeutet sein.

\section{Leitkategorie: Aufgabenbewältigung}

Eine der <inhaltlichen Orientierung> zuzuordnende Kategorie ist die IAufgabenbewältigungll. Diese aktivieren Lehrkräfte meist implizit, wenn sie ihre Praktiken darauf optimieren, dass Lernende in Aufgaben zum Ergebnis kommen, ohne dabei unbedingt etwas zu lernen (Gravemeijer et al. 2016). Sie greift also ausschließlich auf eine <kurzfristige Orientierung > zurück. Sie kann mit der <sozialen Orientierung> verknüpft werden (wie oben) oder isoliert auftreten. Das Transkript zeigt eine typische Äußerung zur IAufgabenbewältigung\| (Transkript 3).

\section{Transkript 3: Rita Sander}

Kontext: Die Lernenden mit Förderschwerpunkt Lernen wollen gerne dasselbe bearbeiten wie die anderen (in der Regel- statt Basisfassung des Materials).

33 Rita $[\ldots]$ wir haben natürlich auch Schüler [im Förderschwerpunkt] Lernen, die mathematisch nicht so schlecht sind $-[\ldots]$ Das ist die Sache des inklusiven Unterrichts. [...] ich habe das Regelheft kopiert und dann habe ich Differenzierung nachgebaut. [..] Die brauchen immer eine Anleitung. [...] Ich erkläre das, wo das Komma hingeht. Ich gebe diese Rechnung vor.

Und schon können die das wieder umsetzen. Das sind so Tipps, die man geben kann und da haben die sich ruckizucki gemeldet. [...]

$<$ soziale Orientierung $>$ \|soziale Teilhabe\| Job Identifizieren/Diagnostizieren Job Schwerpunkte setzen $<$ kurzfristig $><$ Kalkül $>$ $<$ inhaltliche Orientierung $>$ \|Aufgabenbewältigung $\|$ \|soziale Teilhabe\|

Ritas Äußerung in Turn 33 steht im Kontext einer Erzählung, dass sich die Lernenden im Förderschwerpunkt Lernen gerne mit denselben Materialien wie die anderen Lernenden beschäftigen. Rita ist hier <inhaltlich orientiert> und achtet auf die mathematischen Aktivitäten, nicht nur auf ,beschäftigt sein“. Um die angesprochene Ilsoziale Teilhabell zu ermöglichen, differenziert sie die Aufgaben so, dass die Schwächeren sie mit viel ॥Unterstützung\| (,Ich gebe diese Rechnung vor“) lösen können. Den Erfolg misst Rita nicht daran, ob die Schwächeren <langfristig> etwas gelernt haben, sondern ob sie die Aufgaben < kurzfristig > trotz fehlender Voraussetzung bewältigen: ,und da haben die sich ruckizucki gemeldet“. Ritas Unterstützungsstrategie in der Leitkategorie \|Aufgabenbewältigung\| behindert hier das Fördern des inhaltlichen Weiterlernens, da durch die massive Unterstützung kein eigenständiges Den- 
ken mehr möglich ist, ungeachtet ihrer Diagnose, dass sie ,mathematisch nicht so schlecht sind“".

\section{Leitkategorie: Inhaltliche Teilhabe}

Die Leitkategorie Ilinhaltliche Teilhabell aktivieren Lehrkräfte, wenn sie die Beteiligung aller Lernenden am inhaltlichen Lernzuwachs anstreben. Sie basiert auf der Kombination von <inhaltlicher $>$ und $<$ sozialer Orientierung $>$ und wird zum Beispiel mit dem didaktischen Werkzeug der selbstdifferenzierenden Aufgaben realisiert, allerdings oft ohne durch lladaptive Impulsell die Niveaugerechtheit nachzusteuern. Eine typische Äußerung zeigt Transkript 4.

Transkript 4: Jana Nagel

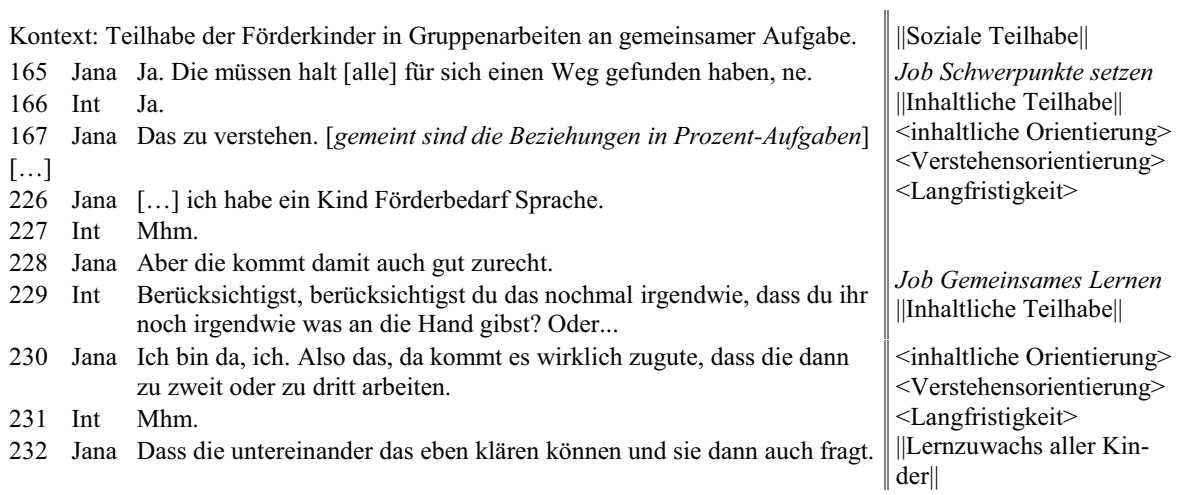

Janas Äußerung ist ein Beispiel, in dem die Kategorie llinhaltliche Teilhabell produktiv genutzt wird. Als Lernziel setzt sie, dass alle Lernenden die Beziehungen in Prozentaufgaben verstehen sollen (,Die müssen halt [alle] für sich einen Weg gefunden haben" in Turn 165) und evaluiert, dass dies auch ihr Förderkind im Förderschwerpunkt Sprache erreicht (,Aber die kommt damit auch gut zurecht.“ in Turn 228). Hier denkt Jana $<$ langfristig $>$ und $<$ verstehensorientiert $>$. Dass dabei nicht nur IIAufgabenbewältigung\| im Blick ist, sondern der ILernzuwachs aller Kinderll, wird an dem Zusatz ,und sie dann auch fragt“ (Turn 232) deutlich. Ein Iladaptiverll Umgang mit verschiedenen IILernstufenll kommt in ihrer Äußerung weder beim Identifizieren und Diagnostizieren noch beim Setzen differenzierter Schwerpunkte vor, daher ist auch das gemeinsame Lernen hier ein gemeinsames Lernen llim Gleichschrittll.

\section{Leitkategorie: Stufengerechte Lernzuwächse}

Die Leitkategorie Ilstufengerechte Lernzuwächsell verknüpft die $<$ langfristige $><$ inhaltliche Orientierung> mit der Kategorie IIAdaptivitätll: Mit dieser Leitkategorie versuchen Lehrkräfte die Lernvoraussetzungen und Lernwege ihrer Lernenden genau zu diagnostizieren, sodass auch Differenzierungen an unterschiedlichen ILernstufungenll ansetzen können. So wird nicht eine möglichst lange linhaltliche Teilhabell an einem gleichschrittigen Programm ermöglicht, sondern Ilstufengerechte Lernzuwächsell. Ein Beispiel zeigt Transkript 5. 


\section{Transkript 5: Ulla Kaiser und Helen Ribert}

Kontext: Worin unterscheiden sich die sonst fünffach differenzierten Arbeitsblätter und wie wird sonst noch gefördert?

201 Ulla Und wenn es dann um Rechnungen geht, nehmen die halt auch ganz oft irgendwie dann noch Plättchen dazu oder irgendwie so, damit die das halt ein bisschen strukturierter darstellen.

202 Helen Weil die Multiplikation ist auch nicht vorhanden.

203 Ulla Ja.

$[\ldots]$

205 Helen Zum größten Teil. Also wir - die LE Schüler sind teilweise Grundschulniveau, zweite, dritte Klasse, ne.

<inhaltliche Orientierung $>$

Job Fördern

||stufengerechte Lernzuwächse\| ||Lernstufung\|

$<$ Verstehensorientierung $>$

Job Identifiz../Diagnostizieren $<$ Verstehensorientierung $>$ $<$ Langfristigkeit $>$

\|stufengerechte Lernzuwächse\|

207 Helen Also da können wir auch nicht viel weiter mit- also Bruchrechnung ist viel zu abstrakt.

$[\ldots]$

267 Ulla Weil mir bringt es nichts, den Zahlenraum zu verändern, wenn die Grundrechenarten nicht da sind.
Job Schwerpunkte setzen

$<$ Verstehensorientierung $>$

$<$ Langfristigkeit $>$

||stufengerechte Lernzuwächse\|

Während Arbeitsblätter mit Fünffach-Differenzierung bei anderen Lehrkräften oft nur nach \|Anforderungsstufung\| differenziert sind, um allen Lernenden die IAufgabenbewältigung\| zu ermöglichen, lässt sich dieses Lehrkraft-Tandem von der Kategorie des Istufengerechten Lernzuwachses\|l leiten: Ulla und Helen legen eine \|Lernstufung\| für das fokussierte Fördern zugrunde, mit der sie die schrittweise Lösung vom graphischen Prozentstreifen im Übergang vom Verstehen zum Kalkül systematisch einplanen (,,nehmen die halt [...] dann [...] Plättchen dazu [...], damit die das halt ein bisschen strukturierter darstellen“ in Turn 201). Dem liegt eine genaue Diagnose zugrunde, welche Lernstufe auf der langfristigen Lernstufung bereits erreicht ist (Turn 202). Die beiden Lehrkräfte identifizieren die IVerstehensgrundlagell Multiplikation als relevante Lernvoraussetzung für den Umgang mit Prozentaufgaben (Turn 202-207) und setzen diese als Schwerpunkt und sogar notwendiges <langfristiges> Lernziel für die Förderkinder (Turn 207, 267, expliziter in nicht abgedruckten Turns). Das fokussierte Fördern zielt darauf ab, die multiplikativen Strukturen durch Plättchen darstellen zu lassen (Turn 201).

\section{Zwischenfazit}

Als erstes Zwischenfazit wird der Zusammenhang der Leitkategorien und ihre Nähe zu den jeweiligen Orientierungen in Abb. 3 aufgezeigt. "Aufgabenbewältigung\| wird in $<$ kurzfristiger Orientierung $>$ fokussiert, llinhaltliche Teilhabell geht bzgl. <langfristiger Orientierung > nicht so weit wie <stufengerechte Lernzuwächse>, daher sind diese weiter oben angeordnet. IISoziale Teilhabell ist nur von sozialer Orientierung getragen, \|inhaltliche Teilhabell und \|Aufgabenbewältigung\| meist von einer Kombination aus <inhaltlicher und sozialer Orientierung $>$. IIStufengerechte Lernzuwächsell allein realisiert nur <inhaltliche Orientierung>, aber keine <soziale>, dafür muss diese Leitkategorie mit z. B. llinhaltlicher Teilhabell verknüpft werden. Im Folgenden werden weitere Zusammenhänge dargelegt. 


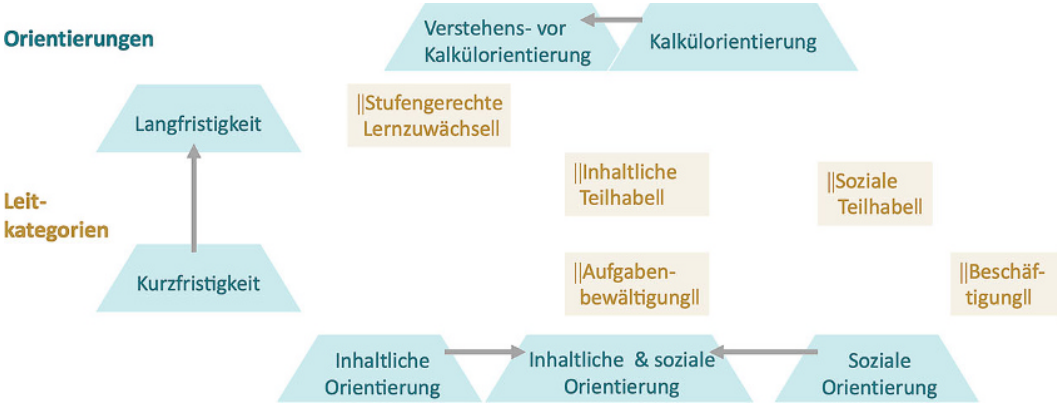

Abb. 3 Fünf Leitkategorien und ihre Nähe zu den Orientierungen. (Bildrechte bei S. Prediger)

\subsection{Vergleich der Praktiken zu den jeweiligen Jobs in verschiedenen Leitkategorien}

Im Folgenden wird anhand der obigen und weiterer Transkriptausschnitte genauer verglichen, wie sich die fünf Leitkategorien in den Praktiken zu unterschiedlichen Jobs ausdrücken.

\subsubsection{Praktiken zum Identifizieren und Diagnostizieren bei verschiedenen Leitkategorien}

Die Praktiken zum Identifizieren und Diagnostizieren unterscheiden sich in den fünf Leitkategorien stark voneinander. Die Leitkategorien bestimmen dabei den Fokus der Diagnosen der Lehrkräfte, wie die Transkriptstellen aus Abschn. 4.1 bereits zeigen:

- Das Identifizieren von Lernvoraussetzungen findet in der Leitkategorie Ilsoziale Teilhabell nicht statt. Stattdessen diagnostizieren Lehrkräfte in der Leitkategorie Ilsoziale Teilhabell meist, inwiefern Lernende mit sonderpädagogischem Förderbedarf Teil der Klassengemeinschaft sind und somit ein Ilsoziales Miteinanderll existiert. In Transkript 1 prüft Quinn in der Leitkategorie, ob sie „sich zugehörig fühlen“ (in Turn 165b).

- In der Leitkategorie ॥Beschäftigung\| werden ebenfalls keine kognitiven Lernvoraussetzungen identifiziert und diagnostiziert. Stattdessen diagnostizieren Lehrkräfte, ob die Lernenden im Unterricht mit fachbezogenen Tätigkeiten beschäftigt sind und nehmen ggf. Ilvolitionale Lernvoraussetzungenll wie Motivation oder metakognitive Regulation in den Blick. In Transkript 2 in Turn 70a merkt Ulla an, dass die schwachen Lernenden in kooperativen Lernformen ,dann einfach gar nichts [machen]" und diagnostiziert somit, ob Lernende sich generell am Unterrichtsgeschehen beteiligen.

- Wenn Lehrkräfte die Bearbeitung von Aufgaben fokussieren, diagnostizieren sie nach der \|Aufgabenbewältigung\|, ob die Lernenden beim Ergebnis angekommen sind. Sie diagnostizieren nicht nur, ob Lernende beschäftigt sind, sondern auch, ob sie die aktuellen Aufgaben, <kurzfristig>, ggf. mithilfe von Unterstützungsmaß- 
nahmen bewältigen. In Transkript 3 zeigt sich dies in der Äußerung „Und schon können die das wieder umsetzen." (Rita, Turn 33).

- Die llinhaltliche Teilhabell lässt sich rekonstruieren, wenn Lehrkräfte inhaltliche Lernstufungen identifizieren und diagnostizieren, inwiefern Lernende nicht nur die "Aufgabe bewältigenll, sondern eine gemeinsame Lernstufe des aktuellen Stoffs auch <verstehen> können, um <langfristig> , gut zurecht [zu kommen]“ (Transkript 4, Jana, Turn 228) und „das zu verstehen“ (Turn 167). Die Leitkategorie taucht häufiger mit $<$ Verstehens- $>$ als mit $<$ Kalkülorientierung $>$ auf.

- Darüber geht das Identifizieren und Diagnostizieren in der Leitkategorie IIStufengerechter Lernzuwächsell hinaus, wenn zusätzlich die für den Inhalt notwendigen ॥Verstehensgrundlagenll und somit Anforderungen des Inhalts an einzelne Lernende lladaptivll identifiziert werden. Helen identifiziert (in Transkript 5, Turn 207) die Multiplikation als Verstehensgrundlage der Prozente und verdeutlicht zugleich ihr Fehlen als Hindernis für das Weiterlernen.

\subsubsection{Praktiken zum Setzen differenzierter Schwerpunkte bei verschiedenen Leitkategorien}

Ausgehend von den dargestellten Diagnosekategorien unterscheiden sich auch die Praktiken in der Anforderungssituation Differenzierte Schwerpunkte setzen, denn mit den Leitkategorien Issoziale Teilhabell, ॥Beschäftigung\| und IAufgabenbewältigung\| fokussieren Lehrkräfte in der Regel keine inhaltlichen Lernziele, in den anderen Leitkategorien hingegen schon. Die Leitkategorien beeinflussen dabei die Differenzierungskategorien:

- In der Leitkategorie Ilsoziale Teilhabell liegt die Schwerpunktsetzung darauf, dass Lernende mit sonderpädagogischem Förderbedarf <langfristig $>$ Teil der $<$ sozialen $>$ Klassengemeinschaft sind. Nur wenn die Leitkategorie mit weiteren kombiniert wird, können inhaltliche Lernziele verfolgt werden.

- In der Leitkategorie \|Beschäftigung\| wird optimiert, dass alle Lernenden (mehr oder weniger) aktiv sind, ohne explizite <inhaltliche Orientierung > auf den Lerngegenstand. Die in Turn 70b von Ulla beschriebene Maßnahme ,[d]ie hat dann wirklich nur mit dem Schüler an dem Material gearbeitet“", setzt den Schwerpunkt, den Lernenden <kurzfristig $>$ wieder zum Arbeiten zu bringen, oft durch Ausgleich der fehlenden Selbstregulation durch äußere Disziplinierung oder Motivation. Eine mögliche Differenzierungspraktik, die in den Unterrichtsvideos des übergreifenden Projekts zu finden ist, ist das IIUnterstützenll durch Hinzunahme von Plättchen, so dass Förderkinder mit irgendetwas „handeln“ können. Sie kommt jedoch in den selbstberichteten Praktiken kaum vor.

- Die Leitkategorie \|Aufgabenbewältigung\| geht darüber hinaus, wenn Lehrkräfte anstreben, dass die <inhaltlichen> Aufgaben mithilfe der angebotenen IIUnterstützungsmaßnahmenll gelöst werden können. Dies realisiert eine $<$ kurzfristige Orientierung > (wie in Transkript 3, Rita, Turn 33 ,da haben die sich ruckizucki gemeldet") ohne einen inhaltlichen ILernzuwachs"l in die Überlegungen einzubeziehen. Differenziert wird durch Anforderungsstufung, z. B. durch mehrere Grade 
der Vorstrukturierung und durch Vorgabe des Lösungswegs, z. B. „Ich gebe diese Rechnung vor.“" (Rita, Turn 33).

- In der Leitkategorie Ilinhaltliche Teilhabell stehen inhaltliche Lernziele im Vordergrund. Dabei wird für alle Lernenden für eine Phase das gleiche Lernziel gesetzt oder eine gewisse Offenheit der Lernziele angelegt, z. B. in Transkript 4, dass ,[alle] für sich einen Weg gefunden haben“ (Jana, Turn 165). Zuweilen sind dafür zusätzliche IIUnterstützungenll (etwa durch Peers wie in Transkript 4, Jana, Turn 230) notwendig. Der Fokussierung inhaltlicher Lernziele liegt meist eine $<$ langfristige $>$ Orientierung zugrunde. Dabei werden zumindest in der Unterrichtseinheit zum Prozentverständnis oft <verstehensorientierte> Lernstufen fokussiert, wie in folgender Äußerung, die offene Lernzielsetzung mit selbstdifferenzierenden Aufgaben anspricht:

77 Uta Wenn es [die Formel] von denen selbst kommt, [...]. Wenn jemand sagt: „Wenn wir das jetzt so und so und so gemacht haben, das ist doch das Gleiche wie das durch das oder was auch immer." Dann finde ich, ist es in Ordnung. Dann kann man es auch [...parallel zum Dreisatz] laufen lassen.

- In der Leitkategorie Ilstufengerechte Lernzuwächsell ist für das Setzen von differenzierten Schwerpunkten die Kategorie IIAdaptivitätll zentral. Die Passung der Lernziele zur aktuellen Lernstufe jedes Lernenden wird kritisch geprüft. Für schwächere Lernende werden dann ggf. nicht nur die aktuellen Lernziele der Unterrichtseinheit fokussiert, sondern auch ihre IVerstehensgrundlagenll vorangehender Jahrgänge, wie z. B. die Multiplikation als Verstehensgrundlage für Hoch- und Runterrechnen bei Prozenten (in Transkript 5, Turn 202). Statt gemeinsamer Lernziele mit differenzierter Unterstützung rücken differenzierte Lernziele ins Zentrum der (offenen oder geschlossenen) Differenzierung.

\subsubsection{Praktiken zum Fördern bei verschiedenen Leitkategorien}

Was eine Förderung erreichen will, hängt eng mit den gesetzten Zielen zusammen. Die Leitkategorien übernehmen hier die Funktion der Förderziele. Wenn (in den ersten drei Leitkategorien) keine inhaltlichen Lernziele fokussiert sind, strebt die Förderung diese auch nicht an:

- In der Leitkategorie Ilsoziale Teilhabell gehen aus den Daten keine selbstberichteten Praktiken zur Anforderungssituation Fördern hervor. Denkbar wären Maßnahmen, die eine Integration eines bislang nicht akzeptierten Jugendlichen in die Klassengemeinschaft stärken können, wie beispielsweise Teambildungsmaßnahmen, o. Ä. um <langfristig > ein <soziales > IMiteinanderll zu ermöglichen.

- Um Lernende im Unterricht zu llbeschäftigenll, ist eine individuelle Förderung im Sinne einer Lernprozessbegleitung zur Motivation oder „Überwachung“ berichtet worden: der „muss dann wirklich ganz klar [...] durchbegleitet werden“ (Transkript 2, Ulla, Turn 70b). 
- Wird die IAufgabenbewältigung\| gestärkt, ohne zugleich Lernziele zu fokussieren, werden in der Regel nicht Förderstrategien, sondern nur Unterstützungsstrategien gewählt. Dass zu viel Unterstützung ohne gleichzeitigen Blick auf Lernziele das Lernen auch verhindern kann, zeigt das Beispiel von Rita in Turn 112:

112 Rita Ich gebe immer die 1x1-Hilfen zu jedem Thema dazu [...]. Die haben an der Seite einen leeren Streifen, wo sie jedes Mal gesagt kriegen: „Schreib dir die Reihe auf, indem du einfach immer wieder plus rechnest."

In anderen Beispielen tauchen \|inhaltliche Teilhabell und \|Aufgabenbewältigung\| kombiniert statt als Gegensatz auf. Dann wird die Stärkung der IAufgabenbewältigung\| i.d.R. so gestaltet, dass auch \|Lernzuwächsell ermöglicht werden.

- In der Leitkategorie llinhaltliche Teilhabell wird Förderung in Praktiken realisiert, in denen Lernende <inhaltlich> dazulernen können, wenn auch nicht unbedingt individuell adaptiv. Im Hinblick auf Förderkinder steht dabei oft die IZugänglichkeit für allell im Vordergrund. Dies erfolgt z. B. durch selbstdifferenzierende Aufgaben oder eine Gesprächsführung, die alle Lernenden wie in folgendem Beispiel inhaltlich einbezieht:

67 Talia [...] Wir haben wiederholt, was bedeutet überhaupt Prozent? Wofür brauchen wir Prozentrechnung? Wiederholung: Stammbrüche, Umwandlung in Dezimalbrüche und umgekehrt in Prozente. Das haben wir zunächst gemacht. Gemeinsam im Plenum, die haben eine Mindmap erstellt. Und dann haben die auch Beispielaufgaben bekommen.

Bei fehlendem Fokus auf IAdaptivitätll wird die gemeinsame Lernstufenerreichung nur global diagnostiziert, nicht individuell. Werden gemeinsame Lernsituationen dagegen auch gezielt selbstdifferenzierend angelegt und mit adaptiven Impulsen versehen, können Ilinhaltliche Teilhabell und IIstufengerechte Lernzuwächsell kombiniert werden.

- Die Förderung in der Leitkategorie der Ilstufengerechten Lernzuwächsell bezeichnen wir in Anlehnung an Prediger (2016) als Praktik des fokussierten Förderns. Der selbstgesetzte Anspruch an fokussierte Förderung ist, dass jedem Lernenden die individuell nächste Lernstufe in einer antizipierten, meist < verstehensorientierten $>$ IILernstufung\| ermöglicht werden soll.

Hierbei wird das Material als Strukturierungsmittel im Sinne der Multiplikation und nicht zur alleinigen \|Aufgabenbewältigung\| herangezogen. Differenzierung kann nicht nur durch Grade der Vorstrukturierung, sondern muss infolgedessen mithilfe von Lernstufung erfolgen, also beispielsweise das Üben des Zählens in Schritten am Prozentstreifen. Das Gelingen des fokussierten Förderns wird durch wiederholtes individuelles Diagnostizieren überprüft. 


\subsubsection{Praktiken zum Orchestrieren gemeinsamen Lernens bei verschiedenen Leitkategorien}

Die rekonstruierten Differenzierungs- und Förderpraktiken in den verschiedenen Leitkategorien haben auch Auswirkungen auf die Praktiken für die Orchestrierung gemeinsamen Lernens:

- Gemeinsames Lernen in der Leitkategorie Ilsoziale Teilhabell fokussiert den Umgang Imiteinanderll, nicht fachliches Lernen. Es könnte sich im <sozialen> Austausch Imiteinanderll ausdrücken, indem Akzeptanz und Toleranz im Prozess (weiter-)entwickelt werden, um <langfristig > das Ziel einer gestärkten Klassengemeinschaft zu erreichen.

- Die Leitkategorie IBBeschäftigung\| adressiert gemeinsames Lernen meist als Sozialform des Klassengesprächs oder der Gruppenarbeit. Innerhalb dieser Sozialformen wird im Lichte der Leitkategorie IBeschäftigung\| optimiert, dass auch in Gruppen- oder Plenumsphasen alle Lernenden aktiv sind, in der Reinform jedoch weiterhin, ohne die inhaltlichen Lernziele zu fokussieren. Frieda beschreibt dies für Lernende mit sonderpädagogischem Förderbedarf:

92 Frieda: [von denen] hat sich vielleicht mal einer, vielleicht auch mal ein zweiter beteiligt.

- Wenn Lehrkräfte sich durch die Leitkategorie \|Aufgabenbewältigung\| leiten lassen, inszenieren sie Prozesse gemeinsamen Lernens z. B. im <inhaltlichen> Austausch über Ergebnisse und im Vorstellen verschiedener $<$ kalkülhafter $>$ Rechenwege, wie etwa Jana:
106-108 Jana Und die Kinder ihre Rechnung vorgestellt haben. [...] Aber das lief dann auch so, dass wir denen mitgeteilt haben und einen Rechenweg geschildert haben, dass die es nachvollziehen konnten und $[\ldots]$ mitgerechnet haben.

Der Austausch ermöglicht den Lernenden ihre Aufgabenbewältigung zu evaluieren und ggf. durch Nachahmen der Lösungswege zu verbessern. Auch die von Lehrkräften oft erwähnten Helfersysteme realisieren sich in reiner IAufgabenbewältigung\| eher als ein Weitersagen der Ergebnisse. Wenn Lehrkräfte die Lernenden sensibilisieren, dass Helfende nicht Lösungen vorwegnehmen, sondern Lernprozesse unterstützen sollen, wird ॥Aufgabenbewältigung\| mit \|linhaltlicher Teilhabell kombiniert.

- Die Leitkategorie der Ilinhaltlichen Teilhabell ist für viele Lehrkräfte beim Sprechen über Praktiken des gemeinsamen Lernens dominant und wird als IMiteinander-Lernenll interpretiert. Das oft formulierte Anliegen, mit allen Lernenden möglichst lang auf der gleichen ILernstufell zu arbeiten, ist dabei von einer impliziten Kategorie der IGleichschrittigkeitll geleitet. Birtes Aussage verdeutlicht eine Auslotung:

396 Birte: Ich gehe mit allen das Heft durch, komplett. [...] Klar peile ich das an, [...] und je nachdem, [...] auf welchem Niveau die unterwegs sind, möchte ich natürlich [...] eine Kompetenz erreichen am Ende. 
- Zudem sind Helfersysteme für viele Lehrkräfte ein wichtiges unterrichtsmethodisches Werkzeug, um heterogenes Arbeitstempo auszugleichen und den schwächeren Lernenden eine längere llinhaltliche Teilhabell zu ermöglichen (wie in Transkript 4, Jana, Turn 230).

- Die Leitkategorie des IIstufengerechten Lernzuwachsesll bedarf weiterer vertiefender Analyse und wird im Folgenden näher beleuchtet.

Vertiefung zum gemeinsamen Lernen mit stufengerechten Lernzuwächsen Die Leitkategorie des Ilstufengerechten Lernzuwachses\|l wird aufgrund der Kategorie "Adaptivitätl von vielen Lehrkräften nur dem individuellen Lernen zugeordnet und als hinderlich für gemeinsames Lernen empfunden. Insbesondere wenn die <inhaltliche Orientierung $>$ exklusiv durch Ilstufengerechten Lernzuwachsll konkretisiert wird, empfinden Lehrkräfte oft einen Konflikt zwischen gemeinsamem Lernen und IAdaptivitätll. Quinns Aussage illustriert das beispielhaft (Transkript 6a).

\section{Transkript 6a: Quinn Tacke}

34 Quinn Dann muss man überlegen, was bespreche ich jetzt. Wenn ich den Teil bespreche, dann können die [einen] nicht mitreden und wenn ich den Teil bespreche, können die [anderen] nicht mitreden.

Job Gemeinsames Lernen in Konflikt zu ||Adaptivität||

Andere Lehrkräfte lösen den vermeintlichen Gegensatz für sich auf, indem sie phasenweise zwischen individualisiertem und gemeinsamem Lernen im Gleichschritt abwechseln, wie z. B. Julia (Transkript 6b).

\section{Transkript 6b: Julia Cohn}

Kontext: Wie besprichst du, wenn Lernende an verschiedenen Aufgaben gearbeitet haben?

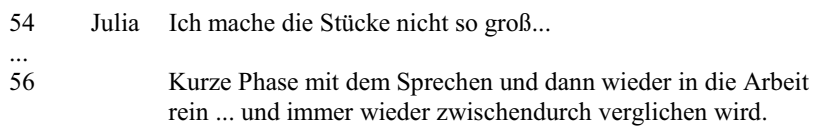

||stufengerechte Lernzuwächse\|

Job Gemeinsames Lernen $<$ soziale Orientierung $>$

Durch kurztaktiges Abwechseln von gemeinsamen und individuellen Phasen versucht Julia (in Turn 56) den Gegensatz zu überwinden. Für das gemeinsame Lernen selbst sehen allerdings weder Quinn (in Transkript 6a) noch Julia (in Transkript 6b) und mit ihnen viele andere Lehrkräfte eine Möglichkeit, innerhalb des IIGleichschritts\| stufengerechtes Lernen zu ermöglichen.

Andere Lehrkräfte dagegen verknüpfen die Leitkategorie Ilstufengerechte Lernzuwächsell mit der Leitkategorie der llinhaltlichen Teilhabell instruktiv. Dadurch können sie <individuelle und soziale Orientierung> vereinen, und zwar durch eine Praktik des Orchestrierens gemeinsamen Lernens auf mehreren Lernstufen: Um aufbauend auf differenzierten Lernzielen gemeinsames Lernen mit je Ilstufengerechten Lernzuwächsenll zu ermöglichen, können diese Lehrkräfte mehrere Lernstufen systematisch aufeinander beziehen, wie in Transkript 6c. 
Transkript 6c: Hanne Ufer

Job Gemeinsames Lernen ||stufengerechte Lernzuwächse\| $<$ inhaltliche Orientierung $>$

||Lernstufung|| ter. Die [Förderkinder] sagten auch sowas wie ,ist das Ganze“, das sagte Emanuel zum Beispiel. Das war richtig gut. Dann kam aber auch jemand, so wie wir uns das gewünscht haben, auf diese Handyladung mit dem Akku. [...] Wir haben das Handy herausgeholt und geguckt mit dem Balken. Und dann kam auch schon „Ah, du hast nur $12 \%$. Das ist aber ganz wenig. " Und das ist für die Inklusionskinder ganz wichtig. Und dann sind wir dazu übergegangen und haben drei verschieden lange Prozentstreifen gehabt [...]. Und da wurden dann eingetragen $40 \%, 60 \%$ und $80 \%$. Da war ganz erstaunlich, wie die Kinder herangegangen sind. [...] Dann ging es wirklich über das Falten. Das war so für die Inklusionskinder eigentlich auch der bessere Weg. Die sind ganz viel über die Hälfte gegangen, über 50 und dann andere noch einmal über 25 . Und viele hatten dann [...] jetzt schon [...] diese Zehnerschritte. Das haben einige Kinder schon gemacht $[\ldots]$.

Hanne berichtet hier von ihrem gemeinsamen, offen-differenzierenden Einstieg in das Thema. Die Situation entwickelt sich aus dem anfänglichen IGleichschrittll in ein ॥Voneinander-Lernenll, bei dem auch Stärkere von Schwächeren lernen. Ihr gelingt es, die Äußerungen von verschiedenen Lernstufen aufeinander zu beziehen, z. B. indem sie den formalbezogenen Ausdruck „Grundwert“ mithilfe von „,das Ganze“ erklären lässt. Die vermeintlich nebensächliche Entdeckung, dass $12 \%$ wenig sind, hebt sie als wichtiges Lernziel für Schwächere hervor.

Hanne kann hier im Igemeinsamen Lernenll zahlreiche Gelegenheiten für IVoneinander-Lernenll auf mehreren ILernstufenll erkennen, vermutlich da sie diese als Lernvoraussetzungen identifiziert hat und deswegen schnell diagnostizieren kann. Gemäß dieser Diagnosen kann sie ad hoc im Gespräch differenzierte Schwerpunkte setzen und Angebote der Lernenden für andere Lernende weiterentwickeln (wie das Vierteln des Streifens, das für Stärkere bereits in Zehnerschritte weiterentwickelt wird).

An diesem Beispiel deutet sich an, dass die Praktik des Orchestrierens gemeinsamen Lernens auf mehreren Lernstufen die komplexeste Praktik ist, die vielfältige Jobs verknüpft und Leitkategorien in fruchtbare Beziehung setzt.

\section{3 Überblick zu individuellen Relevanzsetzungen der Lehrkräfte}

$\mathrm{Zu}$ betonen ist, dass die rekonstruierten Leitkategorien zwar Praktiken charakterisieren, aber keine Typisierung von Lehrkräften sind: Keine Lehrkraft arbeitet nur unter einer einzigen Leitkategorie. Gleichwohl nimmt jede von ihnen andere Schwerpunktsetzungen vor, sowohl in der Gewichtung der Jobs untereinander als auch in den dabei aktivierten Leitkategorien. Dies wird exemplarisch an folgenden Häufigkeitsverteilungen von drei Fokuslehrkräften sichtbar:

- Jana spricht vorrangig den Job Voraussetzungen identifizieren und diagnostizieren an (45-mal). Von den so kodierten Äußerungen wurde in $60 \%$ die Leitkategorie llinhaltliche Teilhabell und in $33 \%$ die IAufgabenbewältigung\| rekonstruiert. Dieselben zwei Kategorien dominieren auch alle anderen Praktiken, wobei den sechs Äußerungen zum gemeinsamen Lernen 4-mal die ॥Aufgabenbewältigung\| 
zugrunde liegt. IIStufengerechte Lernzuwächsell erwähnt sie in insgesamt 99 Äußerungen nur 3-mal, alle beim Setzen differenzierter Schwerpunkte, 7-mal greift sie auf \|soziale Teilhabell und 12-mal auf \|Beschäftigung\| zurück.

- Ulla dagegen verknüpft in ihren 72 kodierten Äußerungen vorrangig Ilinhaltliche Teilhabell und IIstufengerechte Lernzuwächsell. IInhaltliche Teilhabell dominiert in ihren Berichten über die Jobs Voraussetzungen identifizieren (53\%, das sind 24 Äußerungen) und gemeinsames Lernen (100\% von 3 Äußerungen), dagegen dominieren Ilstufengerechte Lernzuwächsell beim Fördern (64\%, 14-mal) und beim Setzen differenzierter Schwerpunkte (53\%, 9-mal). Die anderen Leitkategorien kommen seltener vor: 8-mal greift sie auf Ilsoziale Teilhabell zurück, 3-mal auf \|Beschäftigung\| und 2-mal auf \|Aufgabenbewältigung\|.

- Für Rita wurden nur 52 Äußerungen kodiert, sie variiert jedoch stärker in den Leitkategorien: Beim Voraussetzungen identifizieren und diagnostizieren greift sie zu $54 \%$ (14-mal) auf llinhaltliche Teilhabell zurück und zu je 15\% (4-mal) auf Ilsoziale Teilhabe und Aufgabenbewältigungll. Beim Setzen differenzierter Schwerpunkte werden \|soziale Teilhabell, IAufgabenbewältigung\| und Istufengerechte Lernzuwächsell jeweils in je $29 \%$ der Äußerungen (je 2-mal) leitend, beim Fördern dagegen mit 76\% die IAufgabenbewältigung\|. Gemeinsames Lernen wird nur 2-mal erwähnt, einmal bzgl. Ilsozialer Teilhabell und einmal bzgl. Iinhaltlicher Teilhabell. 4-mal legt sie IBeschäftigung\| als Leitkategorie zugrunde.

Da die Gewichtung im Interview auch von der Gesprächsführung abhängt, ist die Häufigkeitsverteilung mit Vorsicht zu interpretieren. Dennoch geben diese knappen Überblicke erste Annäherungen an die Heterogenität individueller Relevanzsetzungen und an variierende Kohärenz zwischen den Jobs.

Die nochmalige Vergröberung auf die Gesamthäufigkeitsverteilung der insgesamt 1099 kodierten $\ddot{\text { Au}}$ ßerungen aller untersuchten Interviews ist in Abb. 4 dargestellt. Darin zeigt sich, dass der am häufigsten berichtete Job Lernvoraussetzungen identifizieren und diagnostizieren (42\%) ist.

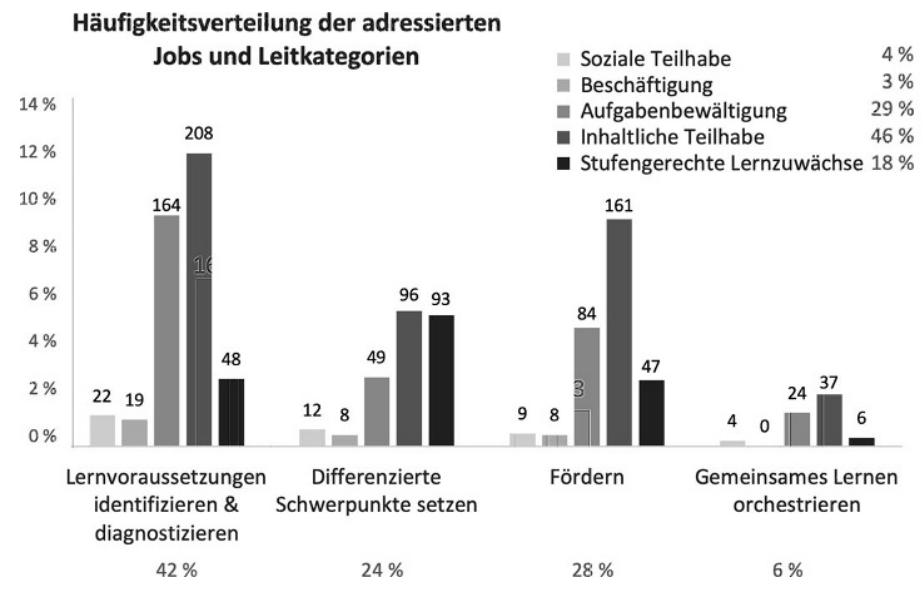

Abb. 4 Häufigkeitsverteilungen der adressierten Jobs und Leitkategorien - Gesamtüberblick für 17 Lehrkräfte. (Bildrechte bei S. Prediger) 
Diagnostiziert wird vorzugsweise bzgl. Ilinhaltlicher Teilhabell, aber auch bzgl. \|Aufgabenbewältigung\| und Istufengerechten Lernzuwächsen\|. Die \|inhaltliche Teilhabell überwiegt auch im Job Setzen differenzierter Schwerpunkte. Die Leitkategorie Ilstufengerechte Lernzuwächsell wurde insbesondere in diesem Job von der Interviewerin gezielt nachgefragt. Die relativ hohe Zahl von 93 Erwähnungen ist also im Gegensatz zu den Anderen nicht selbstinitiiert. IInhaltliche Teilhabell leitet auch die Berichte zum Job Fördern öfter als ॥Aufgabenbewältigung\| und Ilstufengerechte Lernzuwächsell.

Das Orchestrieren gemeinsamen Lernens wird in den Berichten und Reflexionen der Lehrkräfte insgesamt am wenigsten adressiert (6\%), dabei sind llinhaltliche Teilhabell und \|Aufgabenbewältigung\| dominant. Die \|inhaltliche Teilhabell wird meist als ILernen im Gleichschrittll realisiert. Nur in 6 von 1099 kodierten Äußerungen $(0,5 \%)$ wird das gemeinsame Lernen mit der Leitkategorie Ilstufengerechte Lernzuwächsell verknüpft.

IISoziale Teilhabell dagegen wird häufig als erfolgreich etabliert diagnostiziert und dann in den anderen Jobs relativ wenig berücksichtigt. Die eher unproduktive Leitkategorie IBeschäftigung\| leitet das Fördern laut der Selbstberichte der Lehrkräfte nur selten.

\section{Diskussion}

\subsection{Zusammenstellung der rekonstruierten Praktiken zur Bewältigung der Jobs in verschiedenen Leitkategorien}

Oft wurde dokumentiert, dass inklusiver Mathematikunterricht anspruchsvolle Anforderungssituationen an Lehrkräfte stellt, die auf vielfältige und nicht immer produktive Weise bewältigt werden (Korff 2015; Pool Maag und Moser Opitz 2014; Krähenmann et al. 2019). Die vorliegende Interviewstudie leistet einen Beitrag zum Verstehen dieser Beobachtungen, indem sie die selbstberichteten Praktiken der Lehrkräfte bzgl. der zugrundeliegenden Denkkategorien und Orientierungen genauer charakterisiert.

Ausgangspunkt der Studie war die theoriegeleitete Spezifizierung von vier Anforderungssituationen und vier Orientierungen, nach denen die Interviewäußerungen der Lehrkräfte mit 1099 Codes versehen wurden. Dabei zeigten sich zwei interessante Diskrepanzen zwischen didaktischen Unterrichtskonzepten für inklusiven Unterricht und Selbstberichten der Lehrkräfte und damit eine Bestätigung, dass die Berichte nicht durchgängig von sozialer Erwünschtheit geprägt sind:

- Während fachdidaktische und inklusionspädagogische Konzeptionen für inklusiven Mathematikunterricht stark auf das gemeinsame Lernen fokussieren (vgl. Überblick in Kollosche 2019), spielt es in den Berichten der Lehrkräfte nur eine untergeordnete Rolle (s. unten).

- Während fachdidaktische und inklusionspädagogische Konzeptionen für inklusiven Mathematikunterricht gerade die Verknüpfung der Orientierungen als fundamental ansehen (Korff 2015), sind sie bei den Lehrkräften nicht immer produktiv 
verknüpft, sondern zuweilen fragmentiert über verschiedene Jobs oder in gefühltem Widerspruch zueinander.

Es lohnt daher, das Ineinandergreifen der Jobs und Orientierungen genauer zu untersuchen. Empirisch wurden fünf Leitkategorien rekonstruiert, mit denen Lehrkräfte die Orientierungen für sich kombinieren und gewichten: ISoziale Teilhabell, IBeschäftigung\|, \|Aufgabenbewältigung\|, IInhaltliche Teilhabell und IStufengerechte Lernzuwächsell (Abb. 3). Jede dieser Kategorien wurde auch in anderen Studien bereits gefunden:

- ||Beschäftigung\| wurde als häufig auftauchende Leitkategorie des Individualisierens und Differenzierens problematisiert (Martens 2018), sie findet sich in den hier untersuchten Selbstberichten zwar auch wieder, jedoch nur in $3 \%$ der Äußerungen.

- IAufgabenbewältigung\| wurde bereits für Praktiken des Förderns (Gravemeijer et al. 2016) bzw. Diagnostizierens und Förderns von Potentialen (Prediger et al. 2016) empirisch identifiziert und für Differenzierungspraktiken problematisiert (Prediger und von Aufschnaiter 2017). Sie zeigt sich hier in allen inklusionsbezogenen Jobs als oft leitend (in $29 \%$ der Codes).

- IISoziale Teilhabell ist zentral für pädagogische Überlegungen zum inklusiven Unterricht (Wocken 1998). In den Selbstberichten der untersuchten Lehrkräfte wird sie jedoch meist als gelungen diagnostiziert und ist für die weiteren didaktischen Jobs kaum leitend.

- IInhaltliche Teilhabell ist für didaktische Inklusionskonzepte zentral, weil sie <inhaltliche und soziale Orientierungen $>$ verknüpft. In unseren Daten ist sie die dominante Leitkategorie (in $46 \%$ der Codes). Die Analyse der Denkweisen der Lehrkräfte zeigt allerdings wie vielfältig die Leitkategorie verstanden werden kann, so dass weitere Ausdifferenzierungen geboten sind (s. unten).

Nicht die Leitkategorien an sich sind demnach der originäre Beitrag dieser Interviewstudie zur Forschung über Praktiken des inklusiven Unterrichts, sondern die empirische Rekonstruktion, wie sich diese Leitkategorien in den vier Jobs genauer ausprägen. So tauchen die Kategorien z. B. als Diagnosekategorien, als Differenzierungskategorien und auch als Förderziele auf, sowie als (nicht-)leitend für die Orchestrierung gemeinsamen Lernens. Um ihre verschiedenen Ausprägungen zu verstehen, wurde das Zusammenspiel zu den Orientierungen und Werkzeugen empirisch rekonstruiert. Die Befunde aus Abschn. 4.1/4.2 sind in Tab. 1 gebündelt.

Auch wenn eine Quantifizierung von kodierten Äußerungen stets mit Vorsicht zu betrachten ist, geben Häufigkeitsverteilungen einen ersten Aufschluss über heterogene Relevanzsetzungen der Lehrkräfte bzgl. der vier Jobs und fünf Leitkategorien: Jede Lehrkraft hat während des Interviews auf alle fünf Leitkategorien zurückgegriffen, jedoch mit unterschiedlicher Gewichtung für verschiedene Jobs, wie die Fallbeispiele der drei Fokuslehrkräfte in Abschn. 4.3 exemplarisch illustrieren. Die Häufigkeitsverteilungen zu allen 1099 kodierten Äußerungen in Abb. 4 zeigen, dass die 17 befragten Lehrkräfte das Spektrum der Jobs mit verschiedenen Leitkategorien ausschöpfen. Dies legt eine weniger starre Interpretation der Ergebnisse in Tab. 1 nahe. Auf zwei Bereiche wird hier genauer eingegangen, die die Selbstberichte zu 


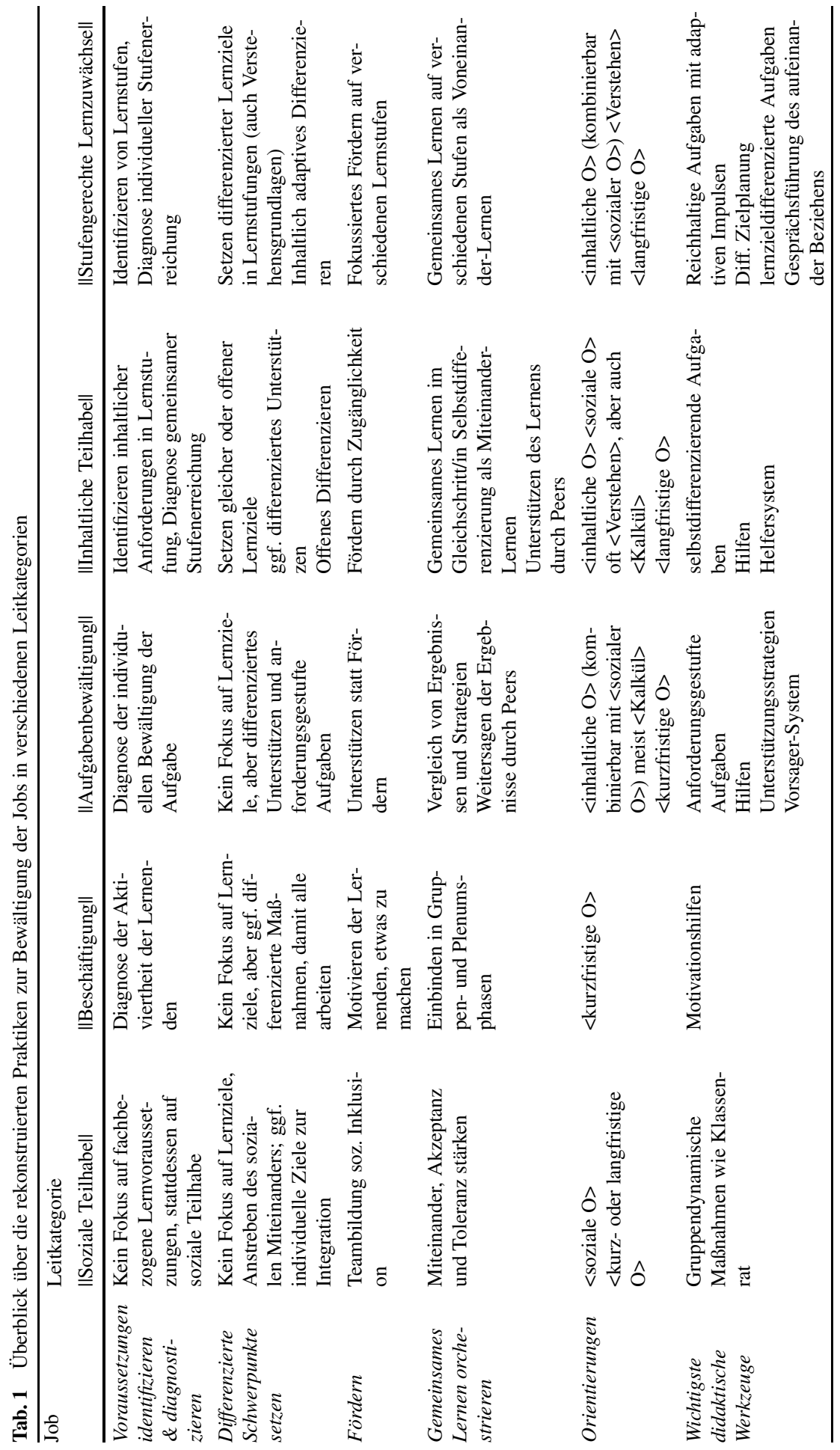


Praktiken über die Balance von individuellem und gemeinsamem Lernen der Lehrkräfte prägen:

Die didaktische Anforderungssituation des Orchestrierens gemeinsamen Lernens wird von den Lehrkräften insgesamt nur in $6 \%$ der Äußerungen angesprochen, also 71-mal. Viermal verbleiben die Lehrkräfte allein in <sozialer Orientierung > mit der Leitkategorie Ilsoziale Teilhabell. In allen anderen Fällen werden <soziale und inhaltliche Orientierung> kombiniert, und zwar in drei verschiedenen Praktiken:

- In der Leitkategorie IAufgabenbewältigung\| geht es um den gemeinsamen Vergleich von Ergebnissen oder um Verkürzung des Helfersystems auf das Vorsagen dieser. Sie wird insgesamt 24-mal beschrieben und liegt oft Praktiken zugrunde, in denen gemeinsam eher an der Sozialform als am gemeinsamen Lernen festgemacht wird.

- In der Leitkategorie llinhaltliche Teilhabell wurden drei Praktiken in 36 Äußerungen berichtet:

- zeitweiliges gemeinsames Lernen im Gleichschritt (meist zu Beginn eines Lernprozesses), was keine differenzierten Lernziele vorsieht und ausloten, wer wann ,,aussteigt“ (Miteinander-Lernen im Gleichschritt)

- gemeinsames Lernen in Selbstdifferenzierung, bei der durch die offenen Aufgaben Bearbeitungen auf verschiedenen Anforderungsstufen möglich sind, jedoch keine differenzierten Lernziele von der Lehrkraft gesetzt werden (MiteinanderLernen in Selbstdifferenzierung)

- Unterstützen in Kleingruppen durch die Peers, das nicht nur auf Ergebnisweitergabe, sondern auch auf Lernzuwächse fokussiert ist (subsidiäres Voneinander-Lernen)

- Die Praktik des gemeinsamen Lernens auf verschiedenen Stufen als VoneinanderLernen in der Leitkategorie Ilstufengerechte Lernzuwächsell kann mit den gleichen selbstdifferenzierenden Aufgaben (oder gezielt stufendifferenzierten Aufgaben) realisiert werden. Die Lehrkraft plant jedoch durch adaptive Impulse oder entsprechende Gesprächsführung stärker, das gemeinsame Lernen auf mehreren Lernstufungen zu orchestrieren. Dies ermöglicht Iinhaltliche Teilhabell und IIstufengerechte Lernzuwächsell zu verknüpfen, setzt jedoch differenziert gesetzte Lernziele voraus. Obwohl es in der Literatur auch für diese Praktiken gut durchgearbeitete Beispiele gibt (Häsel-Weide und Nührenbörger 2017; Prediger und Ademmer 2019), kommt sie nur in 0,5\% der Äußerungen der Lehrkräfte, d. h. 6-mal vor.

Die bei den Lehrkräften rekonstruierte starke Fokussierung auf offene Differenzierungsansätze ohne Berücksichtigung spezifischerer Lernvoraussetzungen hat ein Pendant im Forschungsdiskurs. So zeigt Kollosche (2019) in einem Survey zu 111 deutschsprachigen Forschungsartikeln zum inklusiven Mathematikunterricht, dass 49\% der Artikel auf offene Differenzierungsansätze eingehen, während spezifischere Lernvoraussetzungen kaum berücksichtigt werden.

Rückschauend von dem komplexesten Job gemeinsames Lernen orchestrieren zeigt sich die Bedeutung des Setzens differenzierter Schwerpunkte in der Leitkategorie Ilstufengerechte Lernzuwächsell: Nur, wer für seine Lernenden differenzierte Lernstufungen als Lernziele einplant (dies zeigen in einer schweizerischen Unterrichtsbeobachtungsstudie nur $42 \%$ der Lehrkräfte, Krähenmann et al. 2019), denkt 
daran, diese fokussiert zu fördern und sie gleichzeitig im gemeinsamen Lernen zu orchestrieren.

Das Setzen differenzierter Lernziele erfordert zudem eine <verstehensorientierte> Fokussierung auf Verstehengrundlagen für schwächere Lernende, denn genau im Rückbezug auf Verstehensgrundlagen liegt ein Mehrgewinn für die ganze Klasse (Prediger und Ademmer 2019). Wer dagegen das Setzen differenzierter Schwerpunkte und das Fördern nur in <kurzfristiger Orientierung> auf \|Aufgabenbewältigung\| bezieht, wird keine <langfristig $>$ fruchtbaren Bezugnahmen finden, sondern stärker auf die vielfach in anderen Studien dokumentierten <kalkülorientierten $>$ Unterforderungspraktiken ausweichen (Beswick 2007; Wilhelm et al. 2017; Büscher 2019). Damit erweist sich die Rekonstruktion der Zusammenhänge zwischen den Jobs, Orientierungen und Leitkategorien als Beitrag zur Ausschärfung der Hintergründe typischer Herausforderungen von Lehrkräften.

\subsection{Grenzen der Studie und notwendige Folgestudien}

Die Befunde der Studie sollten stets vor dem Hintergrund ihrer methodischen Grenzen gesehen werden: Mit 17 Lehrkräften ist die Stichprobe relativ klein. Zudem sagt eine Interviewstudie zu selbstberichteten Praktiken mehr über das Denken der Lehrkräfte aus als über ihre tatsächlichen unterrichtlichen Praktiken (Mullens und Gayler 1999). Die rekonstruierten Praktiken und Leitkategorien werden daher im Projekt Matilda durch eine Unterrichtsvideostudie und Analysen der Diskussionen in Fortbildungssitzungen trianguliert und komplementiert.

Die Ergebnisse jeder empirischen Studie sind eingeschränkt durch die spezifischen Kontextbedingungen. In dem vorliegenden Fall betreffen diese insbesondere die Fokussierung auf eine vorgegebene Unterrichtseinheit (Pöhler et al. 2018), in der einige typische Praktiken nicht vorkommen (z. B. zur Individualisierung, vgl. Martens 2018). Sie sind außerdem durch gesetzte Gewichtungen im Gesprächsverlauf bestimmt, wie etwa die Ilstufengerechten Lernzuwächsell, die überwiegend auf gezielte Nachfrage thematisiert wurden. Die Berichte und Reflexionen der Lehrkräfte erfassen daher ggf. ihre Unterrichtspraxis nur partiell, sie zeigen andererseits (trotz einheitlichem Unterrichtsmaterial und einheitlichem Gesprächsleitfaden) eine bemerkenswerte Varianz zwischen Lehrkräften. Folgestudien sollten deshalb zu anderen vorgegebenen Unterrichtseinheiten, mit größerer Stichprobe und ohne Vorgaben die Praktiken und ihre Hintergründe erfassen.

\subsection{Konsequenzen für Fortbildungen: Lernförderliche Anknüpfungspunkte und wichtigste Fortbildungsbedarfe}

Trotz methodischer Begrenzungen lassen sich aus der Interviewstudie Konsequenzen für die inhaltliche Schwerpunktsetzung von Fortbildungen für Lehrkräfte bzgl. inklusiven Mathematikunterrichts ziehen (detaillierter in Prediger et al. 2020), denn sie identifiziert Fortbildungsbedarfe und lernförderliche Anknüpfungspunkte:

- Einen wichtigen Anknüpfungspunkt bildet bei allen untersuchten Lehrkräften die Verknüpfung von <sozialer $>$ und <inhaltlicher Orientierung $>$. Es gilt die Ressour- 
ce zu stärken, dass beide Orientierungen keine Gegensätze bilden müssen, wenn sie in der Leitkategorie der Iinhaltlichen Teilhabell statt \|Aufgabenbewältigung\| realisiert werden.

- Die Leitkategorie \|Aufgabenbewältigung\| kann als Ausgangspunkt für das Bemühen um ein Voranbringen von Lernenden genommen werden. Die Fortbildung sollte allerdings Anlässe schaffen, von einer <kurzfristigen $>$ zu einer $<$ langfristigen> Orientierung auf „Voranbringen“ überzugehen, die die IIstufengerechten Lernzuwächsell mehr ins Zentrum rückt. Ziel ist, dass Lehrkräfte ihre Moderation nicht mehr darauf fokussieren, alle Lernenden zum Aufgabenergebnis zu bringen, sondern allen Lerngelegenheiten geben, um in den Lernstufungen voranzukommen.

- Um differenzierte Schwerpunkte nicht nur in Anforderungsstufungen, sondern in differenzierten Lernzielen zu realisieren (was Krähenmann et al. (2019) als zentrales Qualitätskriterium für inklusiven Unterricht hervorheben), brauchen Lehrkräfte eine tragfähige fachdidaktische Unterstützung, z. B. durch ausgearbeitetes Unterrichtsmaterial. Es sollte <verstehensorientiert> ausgerichtet sein und auch \|Verstehengrundlagenll systematisch integrieren.

- Für gemeinsames Lernen ist die Praktik des Miteinander-Lernens ein guter Ausgangspunkt, auch wenn Lehrkräfte sie vor der Leitkategorie Ilinhaltliche Teilhabell zunächst ohne eigene Zuständigkeit für IIAdaptivitätll deuten. Die Verknüpfung mit Ilstufengerechten Lernzuwächsenll erfordert jedoch den Übergang vom Miteinander-Lernen im Gleichschritt oder von selbstläufig verstandener offener Differenzierung hin zum Voneinander-Lernen auf unterschiedlichen Stufen. Diese für Lehrkräfte anspruchsvollste Praktik braucht in den Fortbildungen mehr Raum, allerdings erst aufbauend auf den zuvor genannten Aspekten.

Danksagung Wir danken Kim-Alexandra Rösike für das Führen einiger Interviews, Katrin Rolka und den anonymen Gutachtenden für wertvolle Rückmeldungen zum Manuskript und unserem Projektteam Jan Kuhl, Christian Büscher, Claudia Ademmer, Claudia Wittich und Imke Pulz für die Kooperation im Projekt.

Förderung Das Projekt Matilda - Mathematik inklusiv lehren lernen wird finanziert vom BMBF (Förderkennzeichen 01NV1704, Projektleitung Susanne Prediger \& Jan Kuhl). Es steht im Forschungskontext des Deutschen Zentrums für Lehrerbildung Mathematik (finanziert durch die Deutsche Telekom-Stiftung).

Funding Open Access funding provided by Projekt DEAL.

Open Access Dieser Artikel wird unter der Creative Commons Namensnennung 4.0 International Lizenz veröffentlicht, welche die Nutzung, Vervielfältigung, Bearbeitung, Verbreitung und Wiedergabe in jeglichem Medium und Format erlaubt, sofern Sie den/die ursprünglichen Autor(en) und die Quelle ordnungsgemäß nennen, einen Link zur Creative Commons Lizenz beifügen und angeben, ob Änderungen vorgenommen wurden.

Die in diesem Artikel enthaltenen Bilder und sonstiges Drittmaterial unterliegen ebenfalls der genannten Creative Commons Lizenz, sofern sich aus der Abbildungslegende nichts anderes ergibt. Sofern das betreffende Material nicht unter der genannten Creative Commons Lizenz steht und die betreffende Handlung nicht nach gesetzlichen Vorschriften erlaubt ist, ist für die oben aufgeführten Weiterverwendungen des Materials die Einwilligung des jeweiligen Rechteinhabers einzuholen.

Weitere Details zur Lizenz entnehmen Sie bitte der Lizenzinformation auf http://creativecommons.org/ licenses/by/4.0/deed.de. 


\section{Literatur}

Beswick, K. (2007). Influencing teachers' beliefs about teaching mathematics for numeracy to students with mathematics learning difficulties. Mathematics Teacher Education and Development, 9, 3-20.

de Boer, A., Pijl, S. J. \& Minnaert, A. (2011). Regular primary schoolteachers' attitudes towards inclusive education: a review of the literature. International Journal of Inclusive Education, 15(3), 331-353.

Boyd, B. \& Bargerhuff, M.E. (2009). Mathematics education and special education: searching for common ground and the implications for teacher education. Mathematics Teacher Education \& Development, $11,54-67$.

Bromme, R. (1992). Der Lehrer als Experte. Bern, Göttingen, Toronto: Huber.

Büscher, C. (2019). Conceptual learning opportunities in teachers' differentiated task designs for inclusive mathematics education. In U. T. Jankvist, M. van den Heuvel-Panhuizen \& M. Veldhuis (Hrsg.), Proceedings of CERME 11 (S. 3604-3611). Utrecht: Freudenthal Group \& ERME.

Gravemeijer, K., Bruin-Muurling, G., Kraemer, J.-M. \& van Stiphout, I. (2016). Shortcomings of mathematics education reform in the Netherlands. Mathematical Thinking and Learning, 18(1), 25-44.

Häsel-Weide, U. \& Nührenbörger, M. (2017). Grundzüge des inklusiven Mathematikunterrichts. In U. Häsel-Weide \& M. Nührenbörger (Hrsg.), Gemeinsam Mathematik lernen - mit allen Kindern rechnen (S. 8-21). Frankfurt a.M.: Grundschulverband.

Hasselhorn, M. \& Gold, A. (2009). Pädagogische Psychologie. Stuttgart: Kohlhammer.

Kollosche, D. (2019). Inclusive mathematics education in the German-speaking community: the politics of the emergence of a research field. In J. Subramanian (Hrsg.), Proceedings of the Tenth International Mathematics Education and Society Conference (S. 514-523). Hyderabad: MES.

Kollosche, D., Marcone, R., Knigge, M., Godoy Penteado, M. \& Skovsmose, O. (Hrsg.). (2019). Inclusive mathematics education. State-of-the-art research from Brazil and Germany. Cham: Springer.

König, J., Gerhard, K., Kaspar, K. \& Melzer, C. (2019). Professionelles Wissen von Lehrkräften zur Inklusion. Pädagogische Rundschau, 73(1), 43-64.

Korff, N. (2015). Inklusiver Mathematikunterricht in der Primarstufe. Baltmannsweiler: Schneider Hohengehren.

Koster, M., Nakken, H., Pijl, S. \& Van Houten, E. (2009). Being part of the peer group: a literature study focusing on the social dimension of inclusion in education. International Journal of Inclusive Education, 13(2), 117-140.

Krähenmann, H., Moser Opitz, E., Schnepel, S. \& Stöckli, M. (2019). Inclusive mathematics instruction: a conceptual framework and selected research results of a video study. In D. Kollosche, R. Marcone, M. Knigge, M. G. Penteado \& O. Skovsmose (Hrsg.), Inclusive mathematics education (S. 179-196). Cham: Springer.

Krajewski, K. \& Ennemoser, M. (2010). Die Berücksichtigung begrenzter Arbeitsgedächtnisressourcen in Unterricht und Lernförderung. In H.-P. Trolldenier, W. Lenhard \& P. Marx (Hrsg.), Brennpunkte der Gedächtnisforschung (S. 337-365). Göttingen: Hogrefe.

Lawrence-Brown, D. (2004). Differentiated instruction: inclusive strategies for standard-based learning that benefit the whole class. American Secondary Education, 32(3), 34-62.

Martens, M. (2018). Individualisieren als unterrichtliche Praxis. In M. Proske \& K. Rabenstein (Hrsg.), Kompendium qualitative Unterrichtsforschung (S. 207-222). Bad Heilbrunn: Klinkhardt.

Mayring, P. (2015). Qualitative content analysis. In A. Bikner-Ahsbahs, C. Knipping \& N. Presmeg (Hrsg.), Approaches to qualitative research in mathematics education (S. 365-380). Dordrecht: Springer.

Melzer, C., Hillenbrand, C., Sprenger, D. \& Hennemann, T. (2015). Aufgaben von Lehrkräften in inklusiven Bildungssystemen - Review internationaler Studien. Erziehungswissenschaft, 26(51), 61-80.

Morris, A. K., Hiebert, J. \& Spitzer, S. M. (2009). Mathematical knowledge for teaching in planning and evaluating instruction? Journal for Research in Mathematics Education, 40(5), 491-529.

Moser, V. \& Kropp, A. (2014). Kompetenzen in inklusiven Settings (KIS) - Vorarbeiten zu einem Kompetenzstrukturmodell sonderpädagogischer Lehrkräfte. Berlin: Humboldt-Universität.

Mullens, J.E. \& Gayler, K. (1999). Measuring classroom instructional processes: using survey and case study field test results to improve item construction. Working Paper No. 1999-08. Washington, DC: National Center for Education Statistics. U.S. Dep. of Education

Pöhler, B., Prediger, S. \& Strucksberg, J. (2018). Prozente verstehen - Inklusive Unterrichtseinheit in Basis- und Regelfassung. Open Educational Ressource sima.dzlm.de/um. Abrufdatum 15.7.2020

Pool Maag, S. \& Moser Opitz, E. (2014). Inklusiver Unterricht - grundsätzliche Fragen und Ergebnisse einer explorativen Studie. Empirische Sonderpädagogik, 6(2), 133-149. 
Prediger, S. (2016). Inklusion im Mathematikunterricht: Forschung und Entwicklung zur fokussierten Förderung statt rein unterrichtsmethodischer Bewältigung. In J. Menthe, D. Höttecke, T. Zabka, M. Hammann \& M. Rothgangel (Hrsg.), Befähigung zu gesellschaftlicher Teilhabe (S. 361-372). Münster: Waxmann.

Prediger, S. (2019). Promoting and investigating teachers' pathways towards expertise for language-responsive mathematics teaching. Mathematics Education Research Journal, 31(4), 367-392.

Prediger, S. (2020). Content-specific theory elements for explaining and enhancing teachers' professional growth in collaborative groups. In H. Borko \& D. Potari (Hrsg.), ICMI Study 25 Conference Proceedings (S. 2-15). Lisbon: ICMI.

Prediger, S. \& Ademmer, C. (2019). Gemeinsam zum Volumen von Quadern: Eine inklusive und sprachsensible Unterrichtsreihe. Mathematik lehren, 214, 13-18.

Prediger, S. \& von Aufschnaiter, C. (2017). Umgang mit heterogenen Lernvoraussetzungen aus fachdidaktischer Perspektive. In T. Bohl, J. Budde \& M. Rieger-Ladich (Hrsg.), Studienbuch Umgang mit Heterogenität in Schule und Unterricht (S. 291-307). Bad Heilbrunn: Kinkhardt.

Prediger, S., Kuhl, J., Büscher, C. \& Buró, S. (2020). Mathematik inklusiv lehren lernen: Entwicklung eines forschungsbasierten interdisziplinären Fortbildungskonzepts. Journal für Psychologie, 28(2), 288-312.

Prediger, S., Schnell, S. \& Rösike, K.-A. (2016). Design research with a focus on content-specific professionalization processes: the case of noticing students' potentials. In S. Zehetmeier, B. RöskenWinter, D. Potari \& M. Ribeiro (Hrsg.), Proceedings of the Third ERME Topic Conference on Mathematics Teaching, Resources and Teacher Professional Development (S. 96-105). Berlin: HumboldtUniversität / HAL Archive.

Scherer, P. \& Moser Opitz, E. (2010). Fördern im Mathematikunterricht der Primarstufe. Heidelberg: Spektrum.

Scherer, P., Beswick, K., DeBlois, L., Healy, L. \& Moser Opitz, E. (2016). Assistance of students with mathematical learning difficulties: how can research support practice? ZDM Mathematics Education, 48(5), 633-649.

Scruggs, T.E. \& Mastropieri, M. A. (1996). Teacher perceptions of mainstreaming/inclusion, 1958-1995: a research synthesis. Exceptional Children, 63(1), 59-74.

Tomlinson, C. A. (2001). How to differentiate instruction in mixed-ability classrooms. Alexandria: Association for Supervision and Curriculum Development.

Wember, F. B. (2013). Herausforderung Inklusion: Ein präventiv orientiertes Modell schulischen Lernens und vier zentrale Bedingungen inklusiven Unterrichts. Zeitschrift für Heilpädagogik, 64(10), 380-388.

Wilhelm, A. G., Munter, C. \& Jackson, K. (2017). Examining relations between teachers' explanations of sources of students' difficulty in mathematics and students' opportunities to learn. The Elementary School Journal, 117(3), 345-370.

Wocken, H. (1998). Gemeinsame Lernsituationen. In A. Hildeschmidt \& I. Schnell (Hrsg.), Integrationspädagogik (S. 37-52). München: Juventa. 